\title{
THE GENERALIZED STAR PRODUCT AND THE FACTORIZATION OF SCATTERING MATRICES ON GRAPHS
}

\author{
V. KOSTRYKIN AND R. SCHRADER*
}

\begin{abstract}
In this article we continue our analysis of Schrödinger operators on arbitrary graphs given as certain Laplace operators. In the present paper we give the proof of the composition rule for the scattering matrices. This composition rule gives the scattering matrix of a graph as a generalized star product of the scattering matrices corresponding to its subgraphs. We perform a detailed analysis of the generalized star product for arbitrary unitary matrices. The relation to the theory of transfer matrices is also discussed.
\end{abstract}

\section{INTRODUCTION}

Potential scattering for one particle Schrödinger operators on the line possesses a remarkable property concerning its (on-shell) scattering matrix given as a $2 \times 2$ matrix-valued function of the energy. Let the potential $V$ be given as the sum of two potentials $V_{1}$ and $V_{2}$ with disjoint support. Then the scattering matrix for $V$ at a given energy is obtained from the two scattering matrices for $V_{1}$ and $V_{2}$ at the same energy by a certain non-linear, noncommutative but associative composition rule. This fact has been discovered independently by several authors (see e.g. [1, 57, 58, 47, 62, 64, 65]) and is an easy consequence of the multiplicative property of the transfer matrix of the Schrödinger equation (see e.g. [41]). It has also been well known in the theory of mesoscopic systems and multichannel conductors (see e.g. [71, 22, 23, 24, 25, 55, 69, 8, 20, 28). In higher space dimensions a similar rule is not known. However, for large separation between the supports of the potentials the scattering matrix at a given energy is asymptotically related to the scattering matrices for $V_{1}$ and $V_{2}$ at the same energy [39, 40].

To the best of our knowledge the composition rule for $2 \times 2$ scattering matrices was first observed in network theory by Redheffer [57, 58], who called it the star product. In our preceding article [42] we extended this result to quasi-one dimensional quantum systems Schrödinger operators on graphs. Such systems are nowadays a subject of intensive study (see e.g. [31, 7, 27, 13, 14]). Some other related works are quoted in [42]. In [63, 48, 66, 67] differential operators with Neuman boundary conditions on "fat graphs" were considered, i.e. on thin domains in $\mathbb{R}^{d}$ which asymptotically shrink to a graph.

There is also a large amount of literature on linear difference operators on graphs. The motivation for the study of such operators comes from the graph theory, where the spectrum of these operators are known to be related to topological properties of the graph [18, 17, 9]. Scattering theory for such operators was developed in [56, 4 ].

In [42] we considered the (continuous) Laplace operator on graphs with an arbitrary number $n$ of open ends (i.e. channels) and with arbitrary boundary conditions at the edges resulting in a self-adjoint operator. We formulated and proved necessary and sufficient conditions for such operators to be self-adjoint. We provided an explicit expression for the resulting unitary $n \times n$ scattering matrix in terms of the boundary conditions, the lengths of the internal lines and

Date: August 14, 2000.

1991 Mathematics Subject Classification. (2000 Revision) Primary 34B45, 34L40; Secondary 47A40, 81U20.

Key words and phrases. Quantum mechanics on graphs, scattering matrix.

PACS Numbers. 03.65.Nk, 72.23.-b, 73.50.-h.

* R.S. supported in part by DFG SFB 288 "Differentialgeometrie und Quantenphysik". 
the given energy. Furthermore, we generalized Redheffer's star product to what we called the generalized star product. This is a non-linear, noncommutative but associative composition rule for unitary matrices not necessarily of equal rank and resulting in a unitary matrix.

Under special circumstances there is an alternative way to describe the generalized star product. Fix $p \geq 1$ and consider the group $\bigcup(p, p)$ with its natural multiplication. As a set this group is isomorphic to some subgroup of $\mathrm{U}(2 p)$. This non-linear set isomorphism is well known in the case $p=1$ (see e.g. [26]) and can be easily generalized to the case of arbitrary $p>1$. Under this isomorphism the multiplication in $\mathrm{U}(p, p)$ induces new nonlinear multiplication $*_{p}$ in this subgroup of $\mathrm{U}(2 p)$, which is our generalized star product. The operation $*_{p}$ can be extended by continuity to the whole $\bigcup(2 p)$. The set $\bigcup(2 p)$ with $*_{p}$ as multiplication is no longer a group, but only a semigroup.

Employing this generalized star product in [42] we provided a formal proof based on the quantum mechanical superposition principle to show how the scattering matrix at the same energy for the whole graph can be obtained from the scattering matrices of two subgraphs obtained by cutting the graph in any way in two. Again for the special case of 2-channel scattering matrices, like potential scattering on the line, this formal argument is well known (see e.g. [20]). In this article we will provide a rigorous proof of this composition rule. It is interesting to note that in this general case the composition rule cannot be reduced to the multiplicative property of the transfer matrix of the Schrödinger equation on the graph.

Such composition rules are important in the study of the electric conduction in multi-terminal mesoscopic systems. By the Landauer-Büttiker theory the electric conduction in mesoscopic systems is directly related to the transmission probability and thus to the scattering matrix [51, 10, 11, 12]. A good introduction into the theory of electronic transport in such systems is given in the book [20] by S. Datta. The formal arguments leading to the composition rule for the scattering matrices are presented on p. $125-126$ of this book.

The composition rules are also very useful in the study of statistical properties of large random or periodic systems. Examples of such systems can be found e.g. in [5], 0, 27]. In [41, 43, 44, 46] we proved that in arbitrary dimensions the scattering phase (or more generally of the spectral shift function) per interaction volume equals (up to a factor $\pi$ ) the difference of the integrated densities of states for the free and interaction theories respectively. In the strictly one-dimensional situation (Schrödinger operators on the line) the Lyapunov exponent is known to be related to the logarithmic density of transmission probability [52, 53, 54, 41]. Due to the Ishii-Pastur-Kotani theorem (see e.g. [19]) the vanishing of the transmission amplitude for almost all values of energy implies localization (i.e. the spectrum must be purely point), see also the related works [71, 22, 23, 24, 25, 55, 69, 8].

Certain Laplace operators on (infinite) periodic graphs were previously considered in [7, 27]. There are also some attempts to consider differential operators on regular graphs with random boundary conditions or on random graphs with deterministic boundary conditions (see e.g. [5]). Some other examples can be found also in [38, Chapter 3]. A difference Laplace operator on the edges of aperiodic tilings was considered in [37]. Such systems provide a main field of application for our composition rule which will be discussed in a forthcoming publication.

The article is organized as follows. In Section 2 we recall the general quantum scattering theory on graphs as given in [42]. In Section 3 we recall the definition of the generalized star product and study some its properties. In particular we show that this product applies to arbitrary unitary matrices. In Section $\emptyset$ we give a rigorous proof of the composition rule for scattering matrices on arbitrary finite graphs. Section 5 is devoted to the special case of graphs having an even number $2 p$ of external lines. If the new graph is obtained by gluing of exactly $p$ lines then it has again $2 p$ external lines. We consider the question whether in this case the composition rule for the scattering matrices can be reduced to the multiplication rule of the corresponding transfer matrices. In general for $p>1$ the answer is negative. We formulate a necessary and 
sufficient condition, which guarantees that the composition rule for the scattering matrices is equivalent to the standard multiplication of transfer matrices.

After completing the work we received the preprint [34] by M. Harmer where among other questions the composition rule for the scattering matrices is also considered. The results there partially recover our Theorem 4.1 below.

We are indebted to P. Kuchment for sending us the preliminary version of the preprint [48] and also for pointing out the works of R. Carlson [13, 14, 15].

\section{The Laplacian on a Graph and its Scattering MatriX}

In this section we will recall the definition of Schrödinger operators on an arbitrary but finite graph and the construction of their scattering matrices [42].

We consider an arbitrary graph $\Gamma$ with a finite number $n \geq 1$ of external and a finite number $m \geq 0$ of internal lines (edges). More precisely this means that outside of a finite domain the graph is isomorphic to the union of $n$ positive half-lines. Any internal line ends at two, not necessary different vertices and has a finite length. We assume that any vertex of $\Gamma$ has non-zero degree, i.e. for any vertex there is at least one edge (internal or external) with which it is incident.

Let the set $\mathcal{E}$ label the external and the set $\mathcal{J}$ the internal lines of the graph. We assume that the sets $\mathcal{E}$ and $\mathcal{J}$ are ordered in an arbitrary but fixed way. To each $e \in \mathcal{E}$ we associate the infinite interval $[0, \infty)$ and to each $i \in \mathcal{J}$ the finite directed interval $\left[0, a_{i}\right]$, where $a_{i}>0$ is the length of this line. With this association the graph becomes directed, such that the initial vertex of an edge of length $a$ corresponds to $x=0$ and the terminal vertex corresponds to $x=a$. The external lines are assumed to be directed in the positive direction of half-lines.

We define the Hilbert space $\mathcal{H}=L^{2}(\Gamma)$ as

$$
\mathcal{H}=\mathcal{H}_{\mathcal{E}} \oplus \mathcal{H}_{\mathcal{J}}, \quad \mathcal{H}_{\varepsilon}=\bigoplus_{e \in \mathcal{E}} \mathcal{H}_{e}, \quad \mathcal{H}_{\mathcal{J}}=\bigoplus_{i \in \mathcal{J}} \mathcal{H}_{i},
$$

where $\mathcal{H}_{e}=L^{2}(0, \infty)$ and $\mathcal{H}_{i}=L^{2}\left(0, a_{i}\right)$. Elements of $\mathcal{H}$ are written as column vectors

$$
\psi=\left(\left\{\psi_{e}\right\}_{e \in \mathcal{E}},\left\{\psi_{i}\right\}_{i \in \mathcal{J}}\right)^{T}=\left(\psi_{\mathcal{E}}, \psi_{\mathcal{J}}\right)^{T}, \quad \psi_{e} \in \mathcal{H}_{e}, \quad \psi_{i} \in \mathcal{H}_{i} .
$$

Similarly we define the Sobolev space $W^{2,2}(\Gamma)$ as

$$
W^{2,2}(\Gamma)=\bigoplus_{e \in \mathcal{E}} W^{2,2}(0, \infty) \oplus \bigoplus_{i \in \mathcal{J}} W^{2,2}\left(0, a_{i}\right),
$$

where $W^{2,2}(0, \infty)$ and $W^{2,2}\left(0, a_{i}\right)$ are the usual Sobolev spaces of square integrable functions whose distributional second derivatives are also square integrable (see e.g. [59]). Let [ ]: $W^{2,2}(\Gamma) \rightarrow$ $\mathbb{C}^{2(n+2 m)}$ be the surjective linear map which associates to each $\psi$ the element [ $\left.\psi\right]$ given as

$$
[\psi]=\left(\begin{array}{c}
\left(\left\{\psi_{e}(0)\right\}_{e \in \mathcal{E}},\left\{\psi_{i}(0)\right\}_{i \in \mathcal{J}},\left\{\psi_{i}\left(a_{i}\right)\right\}_{i \in \mathcal{J}}\right)^{T} \\
\left(\left\{\psi_{e}^{\prime}(0)\right\}_{e \in \mathcal{E}},\left\{\psi_{i}^{\prime}(0)\right\}_{i \in \mathcal{J}},\left\{-\psi_{i}^{\prime}\left(a_{i}\right)\right\}_{i \in \mathcal{J}}\right)^{T}
\end{array}\right)=\left(\begin{array}{l}
\Psi \\
\underline{\psi}^{\prime}
\end{array}\right)
$$

again viewed as a column vector with the same ordering as in $\psi$, i.e. with the ordering given by the ordering of $\mathcal{E}$ and $\mathcal{J}$.

In [42] we showed that for any two $(n+2 m) \times(n+2 m)$ complex matrices $A$ and $B$ with $A B^{*}$ being Hermitian and the $(n+2 m) \times 2(n+2 m)$ matrix $(A, B)$ having maximal rank equal to $n+2 m$, one can define the self-adjoint Laplace operator $\Delta(A, B, \underline{a})$ in $\mathcal{H}$ corresponding to the boundary condition

$$
A \underline{\psi}+B \underline{\psi}^{\prime}=0
$$

Here $\underline{a}=\left(a_{1}, \ldots, a_{m}\right)^{T} \in \mathbb{R}_{+}^{m}, m=\#(\mathcal{J})$. Furthermore, any self-adjoint extension of the Laplacian on the given graph is given by $\Delta(A, B, \underline{a})$ with some matrices $A$ and $B$ satisfying the properties stated above. If $\mathcal{J}=\varnothing$ we simply write $\Delta(A, B)$ instead of $\Delta(A, B, \cdot)$.

Before we turn to the scattering theory for $\Delta(A, B, \underline{a})$ we recall some well-known facts from scattering theory in two Hilbert spaces $\mathfrak{H}_{1}$ and $\mathfrak{H}_{2}$ (see e.g. [60]). Let $H_{1}$ and $H_{2}$ be self-adjoint 
operators in the Hilbert spaces $\mathfrak{H}_{1}$ and $\mathfrak{H}_{2}$ respectively. Let $\mathcal{J}$ be a bounded operator from $\mathfrak{H}_{1}$ into $\mathfrak{H}_{2}$. The two-space wave operators are defined as the strong limits

$$
\Omega^{ \pm}\left(H_{2}, H_{1} ; \mathcal{\partial}\right)=\underset{t \rightarrow \mp \infty}{\operatorname{sim}} e^{i H_{2} t} \mathcal{\partial} e^{-i H_{1} t} P_{\mathrm{ac}}\left(H_{1}\right),
$$

where $P_{\mathrm{ac}}(H)$ denotes the projection onto the absolute continuous subspace of $H$. We consider the sets $\mathfrak{N}_{ \pm}$of elements $g \in \mathfrak{H}_{2}$ for which $\lim _{t \rightarrow \mp \infty}\left\|\partial^{*} e^{-i H_{2} t} P_{\mathrm{ac}}\left(H_{2}\right) g\right\|=0$. The wave operators $\Omega^{ \pm}$are called $\mathcal{J}$-complete if $\mathfrak{H}_{2}=\overline{\operatorname{Ran}\left(\Omega^{ \pm}\right)} \oplus \mathfrak{N}_{ \pm}$. For details we refer e.g. to Chapter 3 of the book [72].

Now we consider a graph $\Gamma_{\mathcal{E}}$ consisting of the external lines of the original graph $\Gamma$. On the graph $\Gamma_{\varepsilon}$ we consider the operator $-\Delta\left(A_{\varepsilon}=0, B_{\varepsilon}=\mathbb{I}\right)$ corresponding to Neumann boundary conditions. Let $\mathcal{J}: \mathcal{H} \rightarrow \mathcal{H}_{\varepsilon}$ be given as $\mathcal{J} \psi=\psi_{\varepsilon}$ according to the notation (2.1). In particular $\mathcal{J}$ is identity if $m=0$. Since we actually deal with finite dimensional perturbations by the Kato-Rosenblum theorem (see e.g. [72, Theorem 6.2.3 and Corollary 6.2.4]) the wave operators $\Omega^{ \pm}\left(-\Delta(A, B, \underline{a}),-\Delta\left(A_{\mathcal{E}}=0, B_{\mathcal{E}}=\mathbb{I}\right) ; \mathcal{J}\right)$ exist and are $\mathcal{J}$-complete. Thus the scattering operator

$$
S\left(-\Delta(A, B, \underline{a}),-\Delta\left(A_{\mathcal{E}}=0, B_{\mathcal{\varepsilon}}=\mathbb{I}\right) ; \mathcal{\partial}\right)=\left(\Omega^{-}\right)^{*} \Omega^{+}: \mathcal{H}_{\mathcal{E}} \rightarrow \mathcal{H}_{\mathcal{E}}
$$

is unitary and its layers $S_{A, B, \underline{a}}(\lambda): \mathbb{C}^{n} \rightarrow \mathbb{C}^{n}$ (in the direct integral representation with respect to $\left.-\Delta\left(A_{\mathcal{E}}=0, B_{\mathcal{E}}=\mathbb{I}\right)\right)$ are also unitary for almost all energies $\lambda \in \mathbb{R}_{+}$.

The resulting scattering matrix is related to the scattering wave function for the operator $-\Delta(A, B, \underline{a})$ at energy $\lambda>0$ as follows. The function $\psi^{k}(\cdot, \lambda)$ indexed by $k \in \mathcal{E}$ and with components

$$
\psi_{j}^{k}(x, \lambda)= \begin{cases}S_{j k}(\lambda) e^{i \sqrt{\lambda} x} & \text { for } j \in \mathcal{E}, j \neq k, \\ e^{-i \sqrt{\lambda} x}+S_{k k}(\lambda) e^{i \sqrt{\lambda} x} & \text { for } j \in \mathcal{E}, j=k, \\ \alpha_{j k}(\lambda) e^{i \sqrt{\lambda} x}+\beta_{j k}(\lambda) e^{-i \sqrt{\lambda} x} & \text { for } j \in \mathcal{J},\end{cases}
$$

solves the Schrödinger equation with the operator $-\Delta(A, B, \underline{a})$ for energy $\lambda>0$.

Recall that in potential scattering for one particle Schrödinger operators the wave operators give precise meaning to the scattering wave functions, i.e. solutions of the Schrödinger equation at positive energy $\lambda>0$. Similarly the wave operators $\Omega^{ \pm}\left(-\Delta(A, B, \underline{a}),-\Delta\left(A_{\varepsilon}=0, B_{\varepsilon}=\mathbb{I}\right)\right.$; $\left.\mathcal{\partial}\right)$ determine the "external part" of the scattering wave function, i.e. $\psi_{j}^{k}(x, \lambda)$ for $j \in \mathcal{E}$. The completness of usual wave operators means that any solution of the Schrödinger equation at energy $\lambda>0$ can be uniquely represented as a superposition of the scattering wave functions. Similarly, in the present context the $\mathcal{J}$-completness of the wave operators $\Omega^{ \pm}\left(-\Delta(A, B, \underline{a}),-\Delta\left(A_{\varepsilon}=0, B_{\varepsilon}=\mathbb{I}\right) ; \mathcal{\partial}\right)$ means that the external part of any solution for the Schrödinger equation with the operator $-\Delta(A, B, \underline{a})$ at energy $\lambda>0$ can uniquely be represented as a linear combination of the external parts of the scattering wave functions (2.4).

The scattering matrix $S_{A, B, \underline{a}}(\lambda)$ as well as the $m \times n$ matrix amplitudes $\alpha_{A, B, \underline{a}}(\lambda)$ and $\beta_{A, B, \underline{a}}(\lambda)$ are determined as solutions to the equation

$$
Z_{A, B, \underline{a}}(\lambda)\left(\begin{array}{c}
S(\lambda) \\
\alpha(\lambda) \\
\beta(\lambda)
\end{array}\right)=-(A-i \sqrt{\lambda} B)\left(\begin{array}{l}
\mathbb{I} \\
0 \\
0
\end{array}\right)
$$

where

$$
Z_{A, B, \underline{a}}(\lambda)=A X_{\underline{a}}(\lambda)+i \sqrt{\lambda} B Y_{\underline{a}}(\lambda)
$$

is the $(n+2 m) \times(n+2 m)$ matrix with

$$
X_{\underline{a}}(\lambda)=\left(\begin{array}{ccc}
\mathbb{I} & 0 & 0 \\
0 & \mathbb{I} & \mathbb{I} \\
0 & e^{i \sqrt{\lambda} \underline{a}} & e^{-i \sqrt{\lambda} \underline{a}}
\end{array}\right)
$$


and

$$
Y_{\underline{a}}(\lambda)=\left(\begin{array}{ccc}
\mathbb{I} & 0 & 0 \\
0 & \mathbb{I} & -\mathbb{I} \\
0 & -e^{i \sqrt{\lambda} \underline{a}} & e^{-i \sqrt{\lambda} \underline{a}}
\end{array}\right) .
$$

Here $e^{ \pm i \sqrt{\lambda} \underline{a}}$ stands for the $m \times m$ diagonal matrix with elements

$$
\left(e^{ \pm i \sqrt{\lambda} \underline{a}}\right)_{j k}=e^{ \pm i \sqrt{\lambda} a_{j}} \delta_{j k}, \quad j, k \in \mathcal{J} .
$$

If det $Z_{A, B, a}(\lambda) \neq 0$ the scattering matrix $S(\lambda)=S_{A, B, \underline{a}}(\lambda)$ as well as the $m \times n$ matrices $\alpha(\lambda)=$ $\alpha_{A, B, \underline{a}}(\lambda)$ and $\beta(\lambda)=\beta_{A, B, a}(\lambda)$ can be uniquely determined by solving the equation $(2.5)$ in the form

$$
\left(\begin{array}{l}
S(\lambda) \\
\alpha(\lambda) \\
\beta(\lambda)
\end{array}\right)=-Z_{A, B, \underline{a}}(\lambda)^{-1}(A-i \sqrt{\lambda} B)\left(\begin{array}{l}
\mathbb{I} \\
0 \\
0
\end{array}\right) .
$$

We denote by $\Sigma_{A, B, \underline{a}}=\left\{\lambda>0 \mid \operatorname{det} Z_{A, B, \underline{a}}(\lambda)=0\right\}$ the set of exceptional points for which $Z_{A, B, \underline{a}}(\lambda)$ is not invertible.

Let $\phi$ be an arbitrary measurable function on the graph $\Gamma$. The subset supp $\phi$ of all edges of the graph $\Gamma$ will be called the support of the function $\phi$ if $\phi \neq 0$ a.e. on supp $\phi$.

In [42] we proved the following

Theorem 2.1. For any boundary condition $(A, B)$ and arbitrary $\underline{a} \in \mathbb{R}_{+}^{m}$ the set $\Sigma_{A, B, \underline{a}}$ equals the set $\sigma_{A, B, \underline{a}}$ of all positive eigenvalues of $-\Delta(A, B, \underline{a})$. This set is discrete and has no finite accumulation points in $\mathbb{R}_{+}$. The eigenfunctions of $-\Delta(A, B, \underline{a})$ have support on the internal lines of the graph. For all $\lambda \in \Sigma_{A, B, \underline{a}}$ the equation (2.5) is still solvable and determines $S_{A, B, \underline{a}}(\lambda)$ uniquely.

Given an arbitrary $n \times n$ unitary matrix $U$ and an energy $\lambda_{0}>0$ we can find boundary conditions $A, B$ defining a self-adjoint operator $-\Delta(A, B)$ on a single-vertex graph (i.e. with $m=0$ ) with $n$ external lines such that the corresponding scattering matrix is given as $S_{A, B}\left(\lambda_{0}\right)=U$. The proof of this fact can be found in [45]. For other inverse problems on graphs we refer to [32, 15].

Recall that by definition the operator $\Delta(A, B, \underline{a})$ is real if it commutes with complex conjugation, i.e. for any $\psi \in \mathcal{D}(\Delta(A, B, \underline{a}))$ the function $\bar{\psi}$ also belongs to $\mathcal{D}(\Delta(A, B, \underline{a}))$ and $\Delta(A, B, \underline{a}) \psi=$ $\overline{\Delta(A, B) \bar{\psi}}$. Equivalently, this means that any $\psi \in \mathcal{D}(\Delta(A, B, \underline{a}))$ (i.e. $\psi \in W^{2,2}(\Gamma)$ satisfying $A \psi+$ $\left.B \psi^{\prime}=0\right)$ also satisfies the equation $\bar{A} \psi+\bar{B} \psi^{\prime}=0$. Thus, $\Delta(A, B, \underline{a})$ is real iff $\operatorname{Ker}(A, B)=\operatorname{Ker}(\bar{A}, \bar{B})$. The last condition is satisfied iff there is an invertible matrix $C_{1}$ such that $A=C_{1} \bar{A}, B=C_{1} \bar{B}$ or alternatively there is an invertible matrix $C_{2}$ such that both $C_{2} A$ and $C_{2} B$ are real. We recall that $\Delta(A, B, \underline{a})=\Delta(C A, C B, \underline{a})$ for any invertible $C$ (see [42] $)$.

In [42, Corollary 3.2] we have proved the following

Theorem 2.2. For arbitrary $\underline{a} \in \mathbb{R}_{+}^{m}, \lambda>0$, and all boundary conditions $A, B$ defining the selfadjoint operator $\Delta(A, B, \underline{a})$

$$
S_{\bar{A}, \bar{B}, \underline{a}}(\lambda)^{T}=S_{A, B, \underline{a}}(\lambda)
$$

In particular, if the operator $\Delta(A, B, \underline{a})$ is real then $S_{A, B, \underline{a}}(\lambda)=S_{A, B, \underline{a}}(\lambda)^{T}$ for all $\lambda>0$.

Here we give an alternative proof.

Proof. From the selfadjointness of the operator $\Delta(A, B, \underline{a})$ it follows that the matrix $A+i \sqrt{\lambda} B$ is invertible for all $\lambda>0$ (see [42]). The relation (2.5) implies that

$$
\left(\begin{array}{c}
S_{A, B, \underline{a}}(\lambda) \\
\alpha_{A, B, \underline{a}}(\lambda) \\
e^{-i \sqrt{\lambda} \underline{a} \beta_{A, B, \underline{a}}(\lambda)}
\end{array}\right)=-(A+i \sqrt{\lambda} B)^{-1}(A-i \sqrt{\lambda} B)\left(\begin{array}{c}
\mathbb{I} \\
\beta_{A, B, \underline{a}}(\lambda) \\
e^{i \sqrt{\lambda} \underline{a} \alpha_{A, B, \underline{a}}(\lambda)}
\end{array}\right) .
$$


Similarly, for the operator $\Delta(\bar{A}, \bar{B}, \underline{a})$ we have

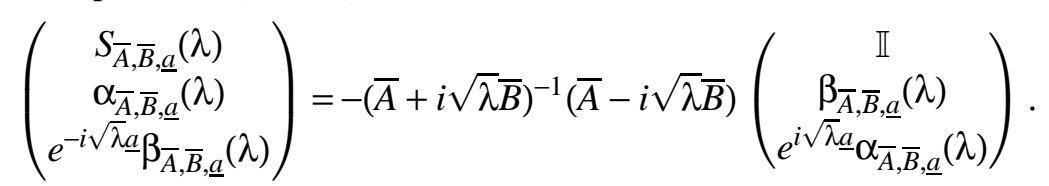

Taking the complex conjugate and multiplying by $(A+i \sqrt{\lambda} B)^{-1}(A-i \sqrt{\lambda} B)$ from the left we obtain

$$
\left(\begin{array}{c}
\frac{\mathbb{I}}{\beta_{\bar{A}, \bar{B}, \underline{a}}(\lambda)} \\
e^{-i \sqrt{\lambda} \underline{a} \alpha_{\bar{A}, \bar{B}, \underline{a}}(\lambda)}
\end{array}\right)=-(A+i \sqrt{\lambda} B)^{-1}(A-i \sqrt{\lambda} B)\left(\begin{array}{c}
\frac{\overline{S_{\bar{A}, \bar{B}, \underline{a}}(\lambda)}}{\alpha_{\bar{A}, \bar{B}, \underline{a}}(\lambda)} \\
e^{i \sqrt{\lambda} \underline{a} \overline{\beta_{\bar{A}, \bar{B}, \underline{a}}(\lambda)}}
\end{array}\right) .
$$

We multiply this relation by $S_{\bar{A}, \bar{B}}(\lambda)^{T}$ from the right and make use of the unitarity of the scattering matrix in the form $\overline{S_{\bar{A}, \bar{B}}(\lambda)} S_{\bar{A}, \bar{B}}(\lambda)^{T}=\mathbb{I}$ thus obtaining

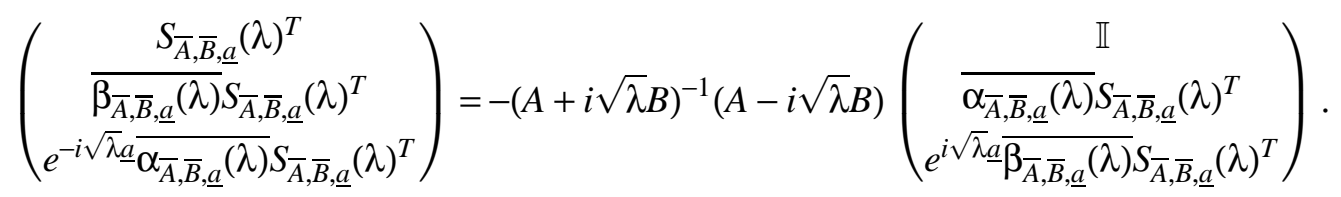

Equivalently, this relation can be written in a form analogous to (2.5),

$$
Z_{A, B, \underline{a}}(\lambda)\left(\begin{array}{c}
S_{\bar{A}, \bar{B}, \underline{a}}(\lambda)^{T} \\
\frac{\beta_{\bar{A}, \bar{B}, \underline{a}}(\lambda) S_{\bar{A}, \bar{B}, \underline{a}}(\lambda)^{T}}{\alpha_{\bar{A}, \bar{B}, \underline{\underline{a}}}(\lambda) S_{\bar{A}, \bar{B}, \underline{a}}(\lambda)^{T}}
\end{array}\right)=-(A-i \sqrt{\lambda} B)\left(\begin{array}{l}
\mathbb{I} \\
0 \\
0
\end{array}\right) .
$$

In [42] we proved that the equation (2.5) has a solution for all $\lambda>0$. If $\lambda>0$ is not an eigenvalue of the operator $\Delta(A, B, \underline{a})$ then it has a unique solution. If $\lambda>0$ is an eigenvalue of $\Delta(A, B, \underline{a})$ this solution is non-unique, but still determines the scattering matrix uniquely. Therefore from comparison of (2.5) and (2.9) the relation (2.8) follows. If $\Delta(A, B, \underline{a})$ is real by the remark preceding the theorem we can choose the matrices $A$ and $B$ to be real. From this and from (2.8) the second claim of the theorem follows.

We note that the comparison of (2.5) with (2.9) also gives the relations

$$
\begin{aligned}
& \alpha_{A, B, \underline{a}}(\lambda)=\overline{\beta_{\bar{A}, \bar{B}, \underline{a}}(\lambda)} S_{A, B, \underline{a}}(\lambda)^{T}, \\
& \beta_{A, B, \underline{a}}(\lambda)=\overline{\alpha_{\bar{A}, \bar{B}, \underline{a}}(\lambda)} S_{A, B, \underline{a}}(\lambda)^{T} .
\end{aligned}
$$

\section{The Generalized Star Product}

Let $U^{(1)}$ and $U^{(2)}$ be arbitrary $n_{1} \times n_{1}$ and $n_{2} \times n_{2}$ unitary matrices respectively. Let $p$ be some integer satisfying $1 \leq p<\left(n_{1}+n_{2}\right) / 2, p \leq n_{j}, j=1,2$, and $V$ be an arbitrary $p \times p$ unitary matrix. We write $U^{(1)}$ and $U^{(2)}$ in a $2 \times 2$-block form

$$
U^{(1)}=\left(\begin{array}{ll}
U_{11}^{(1)} & U_{12}^{(1)} \\
U_{21}^{(1)} & U_{22}^{(1)}
\end{array}\right), \quad U^{(2)}=\left(\begin{array}{cc}
U_{11}^{(2)} & U_{12}^{(2)} \\
U_{21}^{(2)} & U_{22}^{(2)}
\end{array}\right),
$$

where $U_{22}^{(1)}$ and $U_{11}^{(2)}$ are $p \times p$ matrices, $U_{11}^{(1)}$ is an $\left(n_{1}-p\right) \times\left(n_{1}-p\right)$ matrix, $U_{22}^{(2)}$ is an $\left(n_{2}-p\right) \times\left(n_{2}-p\right)$ matrix etc. The unitarity condition for $U^{(1)}$ then reads

$$
\begin{aligned}
& U_{11}^{(1)^{*}} U_{11}^{(1)}+U_{21}^{(1)^{*}} U_{21}^{(1)}=\mathbb{I}, \\
& U_{12}^{(1)^{*}} U_{12}^{(1)}+U_{22}^{(1)^{*}} U_{22}^{(1)}=\mathbb{I}, \\
& U_{11}^{(1)^{*}} U_{12}^{(1)}+U_{21}^{(1)^{*}} U_{22}^{(1)}=0, \\
& U_{12}^{(1)^{*}} U_{11}^{(1)}+U_{22}^{(1)^{*}} U_{21}^{(1)}=0
\end{aligned}
$$


and similarly for $U^{(2)}$.

Definition 3.1. The unitary matrix $U^{(1)}$ is called $V$-compatible with the unitary matrix $U^{(2)}$ if the $p \times p$ matrix $V U_{22}^{(1)} V^{*} U_{11}^{(2)}$ does not have 1 as an eigenvalue. For the case $V=\mathbb{I}$ the matrix $U^{(1)}$ is simply called compatible with $U^{(2)}$ (for given $p \geq 1$ ).

Note that the compatibility of the matrices is not a symmetric relation, i.e. if $U^{(1)}$ is $V$ compatible with $U^{(2)}$ then $U^{(2)}$ need not be $V$-compatible with $U^{(1)}$.

Obviously, if $U^{(1)}$ is $V$-compatible with $U^{(2)}$ then also the matrix $V^{*} U_{11}^{(2)} V U_{22}^{(1)}$ does not have 1 as an eigenvalue. Indeed, let us assume the converse, i.e. let there be non-zero $c \in \mathbb{C}^{p}$ such that $V^{*} U_{11}^{(2)} V U_{22}^{(1)} c=c$ and thus

$$
V U_{22}^{(1)} V^{*} U_{11}^{(2)} V U_{22}^{(1)} c=V U_{22}^{(1)} c .
$$

Since $V U_{22}^{(1)} c \neq 0$ the matrix $V U_{22}^{(1)} V^{*} U_{11}^{(2)}$ has 1 as an eigenvalue, which is a contradiction. From this it also follows that if $U^{(1)}$ is not $V$-compatible with $U^{(2)}$ then the matrix $V^{*} U_{11}^{(2)} V U_{22}^{(1)}$ has 1 as an eigenvalue.

From the unitarity conditions it follows that

$$
\begin{aligned}
& 0 \leq U_{11}^{(1)^{*}} U_{11}^{(1)}=\mathbb{I}-{U_{21}^{(1)^{*}}}^{(1)} \leq \mathbb{I}, \\
& 0 \leq U_{22}^{(1)^{*}} U_{22}^{(1)}=\mathbb{I}-U_{12}^{(1)} U_{12}^{(1)} \leq \mathbb{I},
\end{aligned}
$$

and thus $\left\|U_{11}^{(1)}\right\| \leq 1,\left\|U_{22}^{(1)}\right\| \leq 1$. Similar inequalities hold for $U_{11}^{(2)}$ and $U_{22}^{(2)}$. Therefore $\left\|V U_{22}^{(1)} V^{*} U_{11}^{(2)}\right\| \leq$ 1. Strict inequality holds whenever $\left\|U_{22}^{(1)}\right\|<1$ or $\left\|U_{11}^{(2)}\right\|<1$ and then $U^{(1)}$ is $V$-compatible with $U^{(2)}$ and $U^{(2)}$ is $V$-compatible with $U^{(1)}$ for all unitary $p \times p$ matrices $V$.

In general if $U^{(1)}$ is $V$-compatible with $U^{(2)}$ then it is easy to see that the following $p \times p$ matrices exist:

$$
\begin{aligned}
& K_{1}=\left(\mathbb{I}-V U_{22}^{(1)} V^{*} U_{11}^{(2)}\right)^{-1} V=V\left(\mathbb{I}-U_{22}^{(1)} V^{*} U_{11}^{(2)} V\right)^{-1}, \\
& K_{2}=\left(\mathbb{I}-V^{*} U_{11}^{(2)} V U_{22}^{(1)}\right)^{-1} V^{*}=V^{*}\left(\mathbb{I}-U_{11}^{(2)} V U_{22}^{(1)} V^{*}\right)^{-1} .
\end{aligned}
$$

An easy calculation establishes the following relations

$$
\begin{aligned}
K_{1} & =V+V U_{22}^{(1)} V^{*} U_{11}^{(2)} K_{1}=V+V U_{22}^{(1)} K_{2} U_{11}^{(2)} V \\
& =V+K_{1} U_{22}^{(1)} V^{*} U_{11}^{(2)} V, \\
K_{2} & =V^{*}+V^{*} U_{11}^{(2)} V U_{22}^{(1)} K_{2}=V^{*}+V^{*} U_{11}^{(2)} K_{1} U_{22}^{(1)} V^{*} \\
& =V^{*}+K_{2} U_{11}^{(2)} V U_{22}^{(1)} V^{*} .
\end{aligned}
$$

Note that formally one has the power series expansion

$$
\begin{aligned}
& K_{1}=\sum_{m=0}^{\infty}\left(V U_{22}^{(1)} V^{*} U_{11}^{(2)}\right)^{m} V, \\
& K_{2}=\sum_{m=0}^{\infty}\left(V^{*} U_{11}^{(2)} V U_{22}^{(1)}\right)^{m} V^{*},
\end{aligned}
$$

which is rigorous if $\left\|U_{22}^{(1)}\right\|<1$ or $\left\|U_{11}^{(2)}\right\|<1$. These power series expansions combined with the superposition principle were used in [42] to give a formal proof that the composition rule for scattering matrices was given by the generalized star product.

With these preparations the generalized star product $U=U^{(1)} *_{V} U^{(2)}$ of the unitary matrices $U^{(1)}$ and $U^{(2)}$ is defined as follows. Write the $\left(n_{1}+n_{2}-2 p\right) \times\left(n_{1}+n_{2}-2 p\right)$ matrix $U$ in a $2 \times 2$ block form as

$$
U=\left(\begin{array}{ll}
U_{11} & U_{12} \\
U_{21} & U_{22}
\end{array}\right)
$$


where $U_{11}$ is an $\left(n_{1}-p\right) \times\left(n_{1}-p\right)$ matrix, $U_{22}$ is an $\left(n_{2}-p\right) \times\left(n_{2}-p\right)$ matrix etc. These matrices are now defined as

$$
\begin{aligned}
& U_{11}=U_{11}^{(1)}+U_{12}^{(1)} K_{2} U_{11}^{(2)} V U_{21}^{(1)}, \\
& U_{22}=U_{22}^{(2)}+U_{21}^{(2)} K_{1} U_{22}^{(1)} V^{*} U_{12}^{(2)}, \\
& U_{12}=U_{12}^{(1)} K_{2} U_{12}^{(2)}, \\
& U_{21}=U_{21}^{(2)} K_{1} U_{21}^{(1)} .
\end{aligned}
$$

In particular for an arbitrary $n \times n$ unitary matrix $U$ and all $p$ such that $1 \leq p<n$ the $2 p \times 2 p$ matrices $\mathbb{E}=\left(\begin{array}{ll}0 & \mathbb{I} \\ \mathbb{I} & 0\end{array}\right)$ serve as units for the $*_{V}$-product when $V=\mathbb{I}$,

$$
\left(\begin{array}{ll}
0 & \mathbb{I} \\
\mathbb{I} & 0
\end{array}\right) *_{V} U=U *_{V}\left(\begin{array}{ll}
0 & \mathbb{I} \\
\mathbb{I} & 0
\end{array}\right)=U
$$

Further we will need the following Perron-Frobenius-type result which for the sake of generality will be formulated to cover also the infinite-dimensional case:

Lemma 3.2. Let a compact operator A on a separable Hilbert space $\mathfrak{H}$ be a contraction, i.e. $\|A\| \leq 1$. Suppose that $\lambda=1$ is an eigenvalue of $A$. Then

(i) every $c \in \mathfrak{H}$ such that $A c=c$ also satisfies $A^{*} c=c$ and hence also $A^{*} A c=A A^{*} c=c$,

(ii) the geometric and algebraic multiplicities of the eigenvalue $\lambda=1$ are equal.

Proof. The claim (i) is an easy consequence of the singular values decomposition (see e.g. [35, p. 155]). Thus we have $\operatorname{Ker}(A-1)=\operatorname{Ker}\left(A^{*}-1\right)=(\operatorname{Ran}(A-1))^{\perp}$. The claim (ii) now follows from the fact that the geometric and algebraic multiplicities of an eigenvalue $\lambda$ are unequal iff $\operatorname{Ran}(A-\lambda) \cap \operatorname{Ker}(A-\lambda)$ is non-trivial.

Also we will make use of the following

Lemma 3.3. Let $A$ and $B$ be linear compact operators on a separable Hilbert space $\mathfrak{H}$ such that $\|A\| \leq 1,\|B\| \leq 1$ and $A B b=b$ for some $b \in \mathfrak{H}$. Then $B^{*} B b=b$.

Proof. Without loss of generality we may assume that $\|b\|_{\mathfrak{H}}=1$. By Lemma 3.2

$$
B^{*} A^{*} A B b=b .
$$

Therefore by well-known inequalities for the singular values of compact operators (see e.g. [33]) we have

$$
1 \leq s(A B) \leq s(A) s(B) \leq\|A\|\|B\| \leq 1,
$$

where $s(K)$ denotes the maximal singular value of a compact operator $K$, i.e. the maximal eigenvalue of the self-adjoint non-negative operator $K^{*} K$. This gives $s(A B)=s(A)=s(B)=1$. From $s(A)=s(B)=1$ it follows that $\lambda=1$ is a maximal eigenvalue of $A^{*} A$ and $B^{*} B$. By the min-max principle (see e.g. [61, Theorem XIII.2]) any $c \in \mathfrak{H},\|c\|_{\mathfrak{H}}=1$ maximizing $\left(c, K^{*} K c\right) \leq 1$ satisfies $K^{*} K c=c$. Moreover, if $\left(c, K^{*} K c\right)=1$ with some $\|c\|_{\mathfrak{H}} \leq 1$, then $K^{*} K c=c$ and $\|c\|_{\mathfrak{H}}=1$. Therefore since

$$
\left(B b, A^{*} A B b\right)=\left(b, B^{*} A^{*} A B b\right)=1
$$

we obtain

$$
A^{*} A B b=B b
$$

and $\|B b\|=1$. The relations (3.5) and (3.6) imply that $B^{*} B b=B^{*} A^{*} A B b=b$. 
Suppose now that the unitary matrix $U^{(1)}$ is not $V$-compatible with $U^{(2)}$. In this case the linear subspaces of $\mathbb{C}^{p}$

$$
\begin{aligned}
& \mathcal{C}=\operatorname{Ker}\left(\mathbb{I}-V U_{22}^{(1)} V^{*} U_{11}^{(2)}\right), \quad \widetilde{\mathcal{C}}=\operatorname{Ker}\left(\mathbb{I}-U_{22}^{(1)} V^{*} U_{11}^{(2)} V\right), \\
& \mathcal{B}=\operatorname{Ker}\left(\mathbb{I}-V^{*} U_{11}^{(2)} V U_{22}^{(1)}\right), \quad \widetilde{\mathcal{B}}=\operatorname{Ker}\left(\mathbb{I}-U_{11}^{(2)} V U_{22}^{(1)} V^{*}\right)
\end{aligned}
$$

are nontrivial. By Lemma 3.2 we also have

$$
\begin{aligned}
& \mathcal{C}=\operatorname{Ker}\left(\mathbb{I}-U_{11}^{(2)^{*}} V U_{22}^{(1)^{*}} V^{*}\right), \quad \widetilde{\mathcal{C}}=\operatorname{Ker}\left(\mathbb{I}-V^{*} U_{11}^{(2)^{*}} V U_{22}^{(1)^{*}}\right),
\end{aligned}
$$

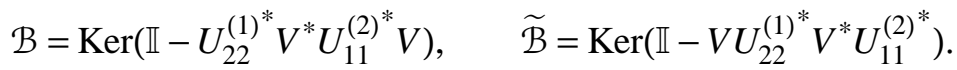

Obviously $\widetilde{\mathcal{C}}=V^{*} \mathcal{C}$ and $\widetilde{\mathcal{B}}=V \mathcal{B}$. Since $V$ is unitary this implies $\operatorname{dim} \widetilde{\mathcal{C}}=\operatorname{dim} \mathcal{C}$ and $\operatorname{dim} \widetilde{\mathcal{B}}=\operatorname{dim} \mathcal{B}$. Furthermore we have

Lemma 3.4. The subspaces $\mathcal{B}$ and $\mathcal{C}$ have equal dimensions, $\operatorname{dim} \mathcal{B}=\operatorname{dim} \mathcal{C}$. Moreover

$$
\begin{aligned}
& \mathcal{B}=\operatorname{lin} \operatorname{span}\left\{V^{*} U_{11}^{(2)} c, \quad c \in \mathcal{C}\right\}=\operatorname{lin} \operatorname{span}\left\{U_{22}^{(1)^{*}} V^{*} c, \quad c \in \mathcal{C}\right\}, \\
& \mathcal{C}=\operatorname{lin} \operatorname{span}\left\{V U_{22}^{(1)} b, \quad b \in \mathcal{B}\right\}=\operatorname{lin} \operatorname{span}\left\{U_{11}^{(2)^{*}} V b, \quad b \in \mathcal{B}\right\}
\end{aligned}
$$

and

$$
\begin{array}{ll}
\widetilde{\mathcal{B}}=\operatorname{lin} \operatorname{span}\left\{U_{11}^{(2)} V \widetilde{c},\right. & \widetilde{c} \in \widetilde{\mathcal{C}}\}=\operatorname{lin} \operatorname{span}\left\{V U_{22}^{(1)^{*}} \widetilde{c}, \quad \widetilde{c} \in \widetilde{\mathcal{C}}\right\}, \\
\widetilde{\mathcal{C}}=\operatorname{lin} \operatorname{span}\left\{U_{22}^{(1)} V^{*} \widetilde{b},\right. & \widetilde{b} \in \widetilde{\mathcal{B}}\}=\operatorname{lin} \operatorname{span}\left\{V^{*} U_{11}^{(2)^{*}} \widetilde{b}, \quad \widetilde{b} \in \widetilde{\mathcal{B}}\right\},
\end{array}
$$

(ii) $U_{21}^{(2)} c=0$ for all $c \in \mathcal{C}$,

(iii) $U_{12}^{(1)} b=0$ for all $b \in \mathcal{B}$,

(iv) $U_{21}^{(1)^{*}} \widetilde{c}=0$ for all $\widetilde{c} \in \widetilde{\mathcal{C}}$,

(v) $U_{12}^{(2)^{*}} \widetilde{b}=0$ for all $\widetilde{b} \in \widetilde{\mathcal{B}}$.

Proof. Let $c_{i} \in \mathbb{C}^{p}, i=1, \ldots, k \leq p(k \geq 1)$ be a (not necessarily orthogonal) basis in $\mathcal{C}$. For all $i=1, \ldots, k$ we have

$$
\left(\mathbb{I}-V U_{22}^{(1)} V^{*} U_{11}^{(2)}\right) c_{i}=0 .
$$

Multiplying these equations by $V^{*} U_{11}^{(2)}$ from the left we obtain

$$
\left(\mathbb{I}-V^{*} U_{11}^{(2)} V U_{22}^{(1)}\right) V^{*} U_{11}^{(2)} c_{i}=0 .
$$

Thus

$$
\text { lin } \operatorname{span}\left\{V^{*} U_{11}^{(2)} c, \quad c \in \mathcal{C}\right\} \subseteq \mathcal{B} .
$$

By Lemma 3.3 it follows from (3.7) that

$$
U_{11}^{(2)^{*}} U_{11}^{(2)} c_{i}=c_{i}, \quad i=1, \ldots, k .
$$

Hence

$$
\operatorname{dim} \operatorname{lin} \operatorname{span}\left\{V^{*} U_{11}^{(2)} c, \quad c \in \mathcal{C}\right\}=\operatorname{dim} \mathcal{C}
$$

and therefore by $(3.8) \operatorname{dim} \mathcal{C} \leq \operatorname{dim} \mathcal{B}$.

Let $b_{i} \in \mathbb{C}^{p}, i=1, \ldots, k^{\prime} \leq p$ be some basis in $\mathcal{B}$. We have

$$
\left(\mathbb{I}-V^{*} U_{11}^{(2)} V U_{22}^{(1)}\right) b_{i}=0
$$

for all $i=1, \ldots, k^{\prime}$. Multiplying these equations by $V U_{22}^{(1)}$ we obtain

$$
\left(\mathbb{I}-V U_{22}^{(1)} V^{*} U_{11}^{(2)}\right) V U_{22}^{(1)} b_{i}=0,
$$


and thus

$$
\text { lin } \operatorname{span}\left\{V U_{22}^{(1)} b, \quad b \in \mathcal{B}\right\} \subseteq \mathcal{C} .
$$

Again by Lemma 3.3 it follows from (3.11) that

$$
U_{22}^{(1)^{*}} U_{22}^{(1)} b_{i}=b_{i}, \quad i=1, \ldots, k^{\prime} .
$$

Thus

$$
\operatorname{dim} \operatorname{lin} \operatorname{span}\left\{V U_{22}^{(1)} b, \quad b \in \mathcal{B}\right\}=\operatorname{dim} \mathcal{B}
$$

and therefore by $(\sqrt{3.12}) \operatorname{dim} \mathcal{B} \leq \operatorname{dim} \mathcal{C}$. So we have proved that $\operatorname{dim} \mathcal{B}=\operatorname{dim} \mathcal{C}$. The inclusion (3.8) and the equality (3.10) imply that

$$
\text { lin } \operatorname{span}\left\{V^{*} U_{11}^{(2)} c, \quad c \in \mathcal{C}\right\}=\mathcal{B} .
$$

The inclusion (3.12) and the equality (3.14) imply that

$$
\text { lin } \operatorname{span}\left\{V U_{22}^{(1)} b, \quad b \in \mathcal{B}\right\}=\mathcal{C} .
$$

The proof of the relations

$$
\begin{aligned}
\mathcal{B} & =\operatorname{lin} \operatorname{span}\left\{U_{22}^{(1)^{*}} V^{*} c, \quad c \in \mathcal{C}\right\}, \\
\mathcal{C} & =\operatorname{lin} \operatorname{span}\left\{U_{11}^{(2)^{*}} V b, \quad b \in \mathcal{B}\right\}
\end{aligned}
$$

is similar and will therefore be omitted.

We turn to the proof of (ii) - (v). By the unitarity of $U^{(2)}$ from (3.9) it follows that $U_{21}^{(2)} U_{21}^{(2)} c_{i}=$ 0 . Since $\operatorname{Ker} A^{*} A=\operatorname{Ker} A$ for any linear operator $A$ we obtain the claim (ii). By the unitarity of

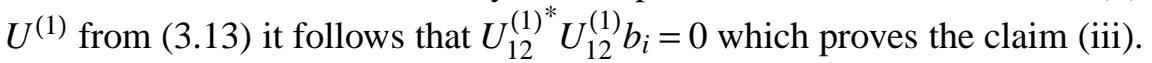

As already noted the vectors $c_{i}$ and $b_{i}$ also satisfy

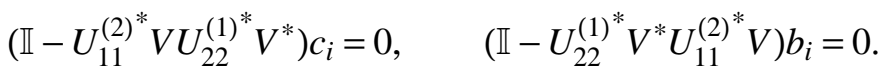

A final application of Lemma 3.3 yields

$$
V U_{22}^{(1)} U_{22}^{(1) *} V^{*} c_{i}=c_{i}, \quad V^{*} U_{11}^{(2)} U_{11}^{(2)^{*}} V b_{i}=b_{i}
$$

which by the unitarity of $U^{(1)}, U^{(2)}$ and $V$ implies (iv) and (v) which completes the proof of the lemma.

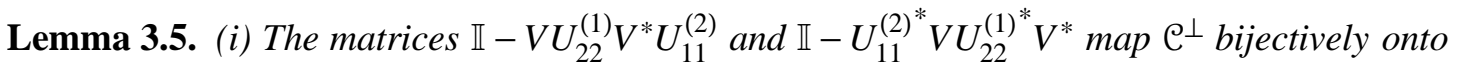
itself,

(ii) the matrices $\mathbb{I}-V^{*} U_{11}^{(2)} V U_{22}^{(1)}$ and $\mathbb{I}-U_{22}^{(1)^{*}} V^{*} U_{11}^{(2)^{*}} V$ map $\mathcal{B}^{\perp}$ bijectively onto itself,

(iii) the matrices $\mathbb{I}-U_{22}^{(1)} V^{*} U_{11}^{(2)} V$ and $\mathbb{I}-V^{*} U_{11}^{(2)^{*}} V U_{22}^{(1)^{*}}$ map $\widetilde{\mathcal{C}}^{\perp}$ bijectively onto itself,

(iv) the matrices $\mathbb{I}-U_{11}^{(2)} V U_{22}^{(1)} V^{*}$ and $\mathbb{I}-V U_{22}^{(1)^{*}} V^{*} U_{11}^{(2)^{*}}$ map $\widetilde{\mathcal{B}}^{\perp}$ bijectively onto itself.

Proof. Since $V$ is unitary by the definitions of $\widetilde{\mathcal{B}}$ and $\widetilde{\mathcal{C}}$ it suffices to prove (i) and (ii). By the definition of $\mathcal{C}$ we have that

$$
\left(c, V U_{22}^{(1)} V^{*} U_{11}^{(2)} c_{\perp}\right)=\left(U_{11}^{(2)^{*}} V U_{22}^{(1)^{*}} V^{*} c, c_{\perp}\right)=\left(c, c_{\perp}\right)=0
$$

for any $c \in \mathcal{C}$ and any $c_{\perp} \in \mathcal{C}^{\perp}$. Thus $\left(\mathbb{I}-V U_{22}^{(1)} V^{*} U_{11}^{(2)}\right) c_{\perp} \in \mathcal{C}^{\perp}$ for all $c_{\perp} \in \mathcal{C}^{\perp}$. Conversely, by Lemma 3.2 for any $c_{\perp} \in \mathcal{C}^{\perp}$ there is a unique $d \in \mathcal{C}^{\perp}$ which satisfies the equation (II$\left.V U_{22}^{(1)} V^{*} U_{11}^{(2)}\right) d=c_{\perp}$. This proves the claim (i). The claim (ii) is proved similarly.

Theorem 3.6. If at least one of the off-diagonal blocks of the matrices $U^{(1)}$ and $U^{(2)}$ (i.e. $U_{12}^{(1)}$, $U_{21}^{(1)}, U_{12}^{(2)}$, or $\left.U_{21}^{(2)}\right)$ is of maximal rank, then the matrix $U^{(1)}$ is $V$-compatible with $U^{(2)}$ for all unitary $p \times p$ matrices $V$. 
Proof. We recall that for $p \leq n_{1} / 2$ the $\left(n_{1}-p\right) \times p$ matrix $U_{12}^{(1)}$ is not of maximal rank $\left(=\min \left\{n_{1}-\right.\right.$ $p, p\})$ iff there is a vector $b \in \mathbb{C}^{p}$ such that $U_{12}^{(1)} b=0$. For $p \geq n_{1} / 2$ the matrix $U_{12}^{(1)}$ is not of maximal rank iff there is a vector $c \in \mathbb{C}^{p}$ such that $U_{12}^{(1)^{*}} c=0$.

Let us suppose that the matrix $U^{(1)}$ is not $V$-compatible with $U^{(2)}$. Then by Lemma 3.4 it follows that all off-diagonal blocks of $U^{(1)}$ and $U^{(2)}$ are not of maximal rank.

Actually we have also the following result. Let a unitary $n \times n$ matrix $U$ be written in the block form

$$
U=\left(\begin{array}{ll}
U_{11} & U_{12} \\
U_{21} & U_{22}
\end{array}\right)
$$

where $U_{11}$ is an $(n-p) \times(n-p)$ matrix, $U_{22}$ is a $p \times p$ matrix etc. with $p$ being an arbitrary integer such that $1 \leq p<n$.

Lemma 3.7. The matrices $U_{12}$ and $U_{21}$ are simultaneously either of maximal rank or not of maximal rank.

Proof. Let us suppose that $p \leq n / 2$. Then the $(n-p) \times p$ matrix $U_{12}$ is not of maximal rank iff there is a non-zero vector $b \in \mathbb{C}^{p}$ such that $U_{12} b=0$. From the unitarity of $U$ it follows that

$$
U_{11}^{*} U_{12}+U_{21}^{*} U_{22}=0, \quad U_{22}^{*} U_{22}+U_{12}^{*} U_{12}=\mathbb{I},
$$

and therefore

$$
U_{21}^{*} U_{22} b=0, \quad U_{22}^{*} U_{22} b=b .
$$

Thus $U_{22} b \neq 0$ and $U_{22} b \in \operatorname{Ker} U_{21}^{*}$. From this it follows that the $(n-p) \times p$ matrix $U_{21}^{*}$ is not of maximal rank, and thus the $p \times(n-p)$ matrix $U_{21}$ is also not of maximal rank.

Now let us suppose that $n>p \geq n / 2$. Then the matrix $U_{12}$ is not of maximal rank iff there is a nontrivial vector $b \in \mathbb{C}^{n-p}$ such that $U_{12}^{*} b=0$. Again from the unitarity we have

$$
U_{21} U_{11}^{*}+U_{22} U_{12}^{*}=0, \quad U_{11} U_{11}^{*}+U_{12} U_{12}^{*}=\mathbb{I},
$$

and therefore

$$
U_{21} U_{11}^{*} b=0, \quad U_{11} U_{11}^{*} b=b .
$$

Thus, the $p \times(n-p)$ matrix $U_{21}$ is not of maximal rank.

We will show now that the $*$-product can be extended to arbitrary, not necessarily $V$-compatible unitary matrices. We will prove that the operators

$$
U_{21}^{(2)} K_{1}=U_{21}^{(2)}\left(\mathbb{I}-V U_{22}^{(1)} V^{*} U_{11}^{(2)}\right)^{-1} V
$$

and

$$
U_{12}^{(1)} K_{2}=U_{12}^{(1)}\left(\mathbb{I}-V^{*} U_{11}^{(2)} V U_{22}^{(1)}\right)^{-1} V^{*}
$$

are actually well-defined. From Lemma 3.5 it follows (see [36, Section I.5.3]) that

$$
P_{\mathcal{C}^{\perp}}\left(\mathbb{I}-V U_{22}^{(1)} V^{*} U_{11}^{(2)}\right)^{-1} \quad \text { and } \quad P_{\mathcal{B}^{\perp}}\left(\mathbb{I}-V^{*} U_{11}^{(2)} V U_{22}^{(1)}\right)^{-1}
$$

are well-defined. From (ii) and (iii) of Lemma 3.4 it follows that $\mathcal{C} \subseteq \operatorname{Ker} U_{21}^{(2)}$ and $\mathcal{B} \subseteq \operatorname{Ker} U_{12}^{(1)}$ and thus

$$
U_{21}^{(2)}\left(\mathbb{I}-V U_{22}^{(1)} V^{*} U_{11}^{(2)}\right)^{-1}=U_{21}^{(2)} P_{\mathcal{C}^{\perp}}\left(\mathbb{I}-V U_{22}^{(1)} V^{*} U_{11}^{(2)}\right)^{-1}
$$

and

$$
U_{12}^{(1)}\left(\mathbb{I}-V^{*} U_{11}^{(2)} V U_{22}^{(1)}\right)^{-1}=U_{12}^{(1)} P_{\mathcal{B}^{\perp}}\left(\mathbb{I}-V^{*} U_{11}^{(2)} V U_{22}^{(1)}\right)^{-1}
$$

are well-defined. Similarly one can show that the operators

$$
U_{21}^{(2)} V\left(\mathbb{I}-U_{22}^{(1)} V^{*} U_{11}^{(2)} V\right)^{-1} \quad \text { and } \quad U_{12}^{(1)} V^{*}\left(\mathbb{I}-U_{11}^{(2)} V U_{22}^{(1)} V^{*}\right)^{-1}
$$


are also well-defined. With this we obtain that the relations (3.4) indeed also define the generalized star product of two non-compatible unitary matrices. Moreover, we have

$$
\begin{array}{rlrl}
U_{21}^{(2)}\left(\mathbb{I}-V U_{22}^{(1)} V^{*} U_{11}^{(2)}\right)^{-1} c & =0, & & U_{12}^{(1)}\left(\mathbb{I}-V^{*} U_{11}^{(2)} V U_{22}^{(1)}\right)^{-1} b=0, \\
U_{21}^{(2)} V\left(\mathbb{I}-U_{22}^{(1)} V^{*} U_{11}^{(2)} V\right)^{-1} \widetilde{c}=0, & & U_{12}^{(1)} V^{*}\left(\mathbb{I}-U_{11}^{(2)} V U_{22}^{(1)} V^{*}\right)^{-1} \widetilde{b}=0
\end{array}
$$

for all $c \in \mathcal{C}, b \in \mathcal{B}, \widetilde{c} \in \widetilde{\mathcal{C}}$, and $\widetilde{b} \in \widetilde{\mathcal{B}}$.

Theorem 3.8. For arbitrary unitary matrices $U^{(1)}, U^{(2)}$, and $V$ the matrix $U=U^{(1)} *_{V} U^{(2)}$ is unitary.

This theorem was proved in Appendix $\mathrm{C}$ of [42] in the case when $U^{(1)}$ is $V$-compatible with $U^{(2)}$. For the general case the proof is given in Appendix A below.

Analogously one can prove associativity of the generalized star product. More precisely let $U^{(3)}$ be a unitary $n_{3} \times n_{3}$ and $V^{\prime}$ a unitary $p^{\prime} \times p^{\prime}$ matrix with $p^{\prime} \leq n_{2}, p^{\prime} \leq n_{3}$. If $p+p^{\prime} \leq n_{1}$, then

$$
U^{(1)} *_{V}\left(U^{(2)} *_{V^{\prime}} U^{(3)}\right)=\left(U^{(1)} *_{V} U^{(2)}\right) *_{V^{\prime}} U^{(3)}
$$

holds.

Theorem 3.9. The generalized star product is a continuous operation in each of its two arguments, i.e. for any unitary matrices $U^{(1)}, U^{(2)}, U^{(3)}$, and $V$ such that $U^{(2)}$ and $U^{(3)}$ have equal size there is a constant $C>0$ depending on $U^{(1)}$ and $V$ only such that

$$
\left\|U^{(1)} *_{V} U^{(2)}-U^{(1)} *_{V} U^{(3)}\right\| \leq C\left\|U^{(2)}-U^{(3)}\right\|,
$$

where the norm $\|\cdot\|$ is an arbitrary matrix norm. A similar estimate holds with respect to the first argument.

In [42] we proved that the scattering matrix of a self-adjoint Laplacian on an arbitrary graph is a continuous function of $\lambda>0$. Theorem 3.9 together with the composition rule given in Section $\emptyset$ below allows to give an alternative proof of this fact. We will not dwell on the details here.

In the sequel we will use the notion of the Moore-Penrose pseudoinverse (see e.g. [70]). Recall that for any (not necessarily square) matrix $M$ its pseudoinverse $M^{\star}$ is uniquely defined by the Penrose equations

$$
\begin{aligned}
M M^{\star} M=M, & M^{\star} M M^{\star}=M^{\star}, \\
\left(M^{\star} M\right)^{*}=M^{\star} M, & \left(M M^{\star}\right)^{*}=M M^{\star} .
\end{aligned}
$$

One also has

$$
\begin{aligned}
M^{\star *} & =M^{* \star}, \\
\operatorname{Ran} M^{\star} & =\operatorname{Ran} M^{*}, \\
\operatorname{Ker} M^{\star} & =\operatorname{Ker} M^{*},
\end{aligned}
$$

and $M M^{\star}=P_{\operatorname{Ran} M}, M^{\star} M=P_{\operatorname{Ran} M^{*}}$, where $P_{\mathcal{H}}$ denotes the orthogonal projection onto the linear subspace $\mathcal{H}$. Moreover $0^{\star}=0$. If $M$ is a square matrix of maximal rank then $M^{\star}=M^{-1}$.

Let $U$ again be an arbitrary unitary $n \times n$ matrix written in the block form with some $1 \leq p<n$.

Lemma 3.10. If $\operatorname{Ker} U_{12}=\{0\}$ then $\operatorname{Ker}\left(U_{21}-U_{22} U_{12}^{\star} U_{11}\right)^{*}=\{0\}$.

Proof. Assume the converse, i.e. let there be $c \in \mathbb{C}^{p}, c \neq 0$ such that

$$
\left(U_{21}-U_{22} U_{12}^{\star} U_{11}\right)^{*} c=0,
$$

or, equivalently,

$$
U_{21}^{*} c-U_{11}^{*} U_{12}^{\star} U_{22}^{*} c=0
$$


We multiply this equation by $U_{21}$ from the left and use the unitarity of $U$ which in particular implies

$$
U_{21} U_{21}^{*}+U_{22} U_{22}^{*}=\mathbb{I} \text {. }
$$

This yields

$$
c-U_{22} U_{22}^{*} c-U_{21} U_{11}^{*} U_{12}^{\star}{ }^{*} U_{22}^{*} c=0 .
$$

Again by unitarity we have $U_{21} U_{11}^{*}=-U_{22} U_{12}^{*}$. Recall that

$$
U_{12}^{*} U_{12}^{\star}{ }^{*}=\left(U_{12}^{\star} U_{12}\right)^{*}=\left(\mathbb{I}-P_{\operatorname{Ker} U_{12}}\right)^{*}=\mathbb{I}
$$

by the hypothesis of the lemma. Thus, from (3.16) it follows that $c=0$.

Now we turn to a discussion of the inverse of a unitary $2 p \times 2 p$ matrix $U$ with respect to the generalized star product $*_{p}$, i.e. the existence of the unitary matrices $U^{L}$ and $U^{R}$ such that

$$
U^{L} *_{p} U=U *_{p} U^{R}=\mathbb{E},
$$

where $\mathbb{E}$ is the $2 p \times 2 p$ matrix $\left(\begin{array}{ll}0 & \mathbb{I} \\ \mathbb{I} & 0\end{array}\right)$ (in the $p \times p$ block notation). We will not discuss general necessary and sufficient conditions for the existence of $U^{L}$ and $U^{R}$, but simply restrict ourselves to a special case. We will prove

Theorem 3.11. Let $U$ be an arbitrary $2 p \times 2 p(p \geq 1)$ unitary matrix. Let at least one of the $p \times p$ matrices $U_{12}$ and $U_{21}$ be of maximal rank $(=p)$. Then there exists a unique unitary $2 p \times 2 p$ matrix $U^{\prime}$ such that

$$
U^{\prime} *_{p} U=U *_{p} U^{\prime}=\mathbb{E} .
$$

Proof. By Lemma 3.7 both matrices $U_{12}$ and $U_{21}$ have maximal rank. We will discuss only the second of the relations (3.17). In block notation this relation has the form

$$
\begin{aligned}
U_{11}+U_{12}\left(\mathbb{I}-U_{11}^{\prime} U_{22}\right)^{-1} U_{11}^{\prime} U_{21} & =0, \\
U_{22}^{\prime}+U_{21}^{\prime}\left(\mathbb{I}-U_{22} U_{11}^{\prime}\right)^{-1} U_{22} U_{12}^{\prime} & =0, \\
U_{12}\left(\mathbb{I}-U_{11}^{\prime} U_{22}\right)^{-1} U_{12}^{\prime} & =\mathbb{I}, \\
U_{21}^{\prime}\left(\mathbb{I}-U_{22} U_{11}^{\prime}\right)^{-1} U_{21} & =\mathbb{I} .
\end{aligned}
$$

By Theorem 3.6 the matrix $U$ must be compatible with $U^{\prime}$ such that $\left(\mathbb{I}-U_{11}^{\prime} U_{22}\right)^{-1}$ and $(\mathbb{I}-$ $\left.U_{22} U_{11}^{\prime}\right)^{-1}$ are both well defined. We multiply the first of the relations (3.18) by $U_{12}^{-1}$ from the left. Next we multiply the resulting expression by $\mathbb{I}-U_{11}^{\prime} U_{22}$ thus obtaining

$$
U_{11}^{\prime}\left(U_{21}-U_{22} U_{12}^{-1} U_{11}\right)=-U_{12}^{-1} U_{11} \text {. }
$$

By Lemma 3.10 we have that $U_{21}-U_{22} U_{12}^{-1} U_{11}$ is invertible and thus

$$
U_{11}^{\prime}=-U_{12}^{-1} U_{11}\left(U_{21}-U_{22} U_{12}^{-1} U_{11}\right)^{-1} \text {. }
$$

From the third relation in (3.18) we obtain

$$
U_{12}^{\prime}=\left(\mathbb{I}-U_{11}^{\prime} U_{22}\right) U_{12}^{-1}=U_{12}^{-1}+U_{12}^{-1} U_{11}\left(U_{21}-U_{22} U_{12}^{-1} U_{11}\right)^{-1} U_{22} U_{12}^{-1} .
$$

The fourth relation in (3.18) gives

$$
U_{21}^{\prime}=U_{21}^{-1}\left(\mathbb{I}-U_{22} U_{11}^{\prime}\right)=\left(U_{21}-U_{22} U_{12}^{-1} U_{11}\right)^{-1} .
$$

The second relation in (3.18) determines $U_{22}^{\prime}$.

It remains to prove that $U^{\prime}$ is unitary. By the unitarity of the matrix $U$ we have

$$
\left(U_{21}^{*}-U_{11}^{*} U_{12}^{*}{ }^{-1} U_{22}^{*}\right)\left(U_{21}-U_{22} U_{12}^{-1} U_{11}\right)=\mathbb{I}+U_{11}^{*} U_{12}^{*-1} U_{12}^{-1} U_{11} .
$$


Therefore

$$
\left(U_{21}^{*}-U_{11}^{*} U_{12}^{*-1} U_{22}^{*}\right)^{-1}\left[\mathbb{I}+U_{11}^{*} U_{12}^{*-1} U_{12}^{-1} U_{11}\right]\left(U_{21}-U_{22} U_{12}^{-1} U_{11}\right)^{-1}=\mathbb{I},
$$

and thus by (3.19) and (3.20) we obtain

$$
U_{11}^{\prime *} U_{11}^{\prime}+U_{21}^{\prime *} U_{21}^{\prime}=\mathbb{I} \text {. }
$$

The relations $U_{12}^{\prime}{ }^{*} U_{12}^{\prime}+U_{22}^{\prime}{ }^{*} U_{22}^{\prime}=\mathbb{I}, U_{11}^{\prime}{ }^{*} U_{12}^{\prime}+U_{21}^{\prime}{ }^{*} U_{22}^{\prime}=0$, and $U_{12}^{\prime}{ }^{*} U_{11}^{\prime}+U_{22}^{\prime}{ }^{*} U_{21}^{\prime}=0$ can be proved similarly.

The left inverse is constructed similarly and by means of the obvious relation

$$
\left(U_{21}-U_{22} U_{12}^{-1} U_{11}\right)^{-1} U_{22} U_{12}^{-1}=U_{21}^{-1} U_{22}\left(U_{21}-U_{22} U_{12}^{-1} U_{11}\right)^{-1} U_{22} U_{12}^{-1}
$$

is easily shown to be equal to the right inverse.

Corollary 3.12. Let $\mathrm{G}$ be the set of all $2 p \times 2 p$ unitary matrices with $p \times p$ blocks $U_{12}$ and $U_{21}$ both being of maximal rank, endowed with the generalized star product $*_{p}$ as a multiplication and $\mathbb{E}$ as a unit. Then $\mathrm{G}$ is a group isomorphic to $\mathrm{U}(p, p)$.

The proof will follow from the arguments given in Section 5. In particular, the group isomorphism between $\mathrm{G}$ and $\mathrm{U}(p, p)$ is given by the formulas (5.9) and (5.13) below. We note that this isomorphism generalizes the well-known set isomorphism between the group $\mathrm{SU}(1,1)$ and a subgroup of $\mathrm{SU}(2)$.

The set of all $2 p \times 2 p$ unitary matrices endowed with the generalized star product $*_{p}$ as a multiplication and $\mathbb{E}$ as a unit is no longer a group but only a semigroup.

\section{Composition Rule For the ScAtTering Matrices}

Now we apply the generalized star product to prove the composition rule for the scattering matrices on graphs. For this we only need the special case $V=\mathbb{I}_{p}$, the $p \times p$ unit matrix, and so we introduce the notation $*_{p}:=*_{V=\mathbb{I}_{p}}$. Let $\Gamma_{1}$ and $\Gamma_{2}$ be two graphs with $n_{1} \geq 1$ and $n_{2} \geq 1$ external lines, respectively, labeled by $\mathcal{E}_{1}$ and $\mathcal{E}_{2}$, i.e. $\#\left(\mathcal{E}_{1}\right)=n_{1}, \#\left(\mathcal{E}_{2}\right)=n_{2}$ and an arbitrary number of internal lines with given fixed lengths (see Fig. 1). Furthermore at all vertices we have local boundary conditions giving Laplace operators $\Delta\left(\Gamma_{1}\right)$ on $\Gamma_{1}$ and $\Delta\left(\Gamma_{2}\right)$ on $\Gamma_{2}$ and the scattering matrices $S_{1}(\lambda)$ and $S_{2}(\lambda)$. Let now $\mathcal{E}_{1}^{0}$ and $\mathcal{E}_{2}^{0}$ be subsets of $\mathcal{E}_{1}$ and $\mathcal{E}_{2}$ respectively having an equal number $1 \leq p \leq \min \left\{n_{1}, n_{2}\right\}$ of elements. Also let $\varphi_{0}: \mathcal{E}_{1}^{0} \rightarrow \mathcal{E}_{2}^{0}$ be a one-to-one map. Finally to each $k \in \mathcal{E}_{1}^{0}$ we associate a number $a_{k}>0$. With these data we can now form a graph $\Gamma$ by connecting the external line $k \in \mathcal{E}_{1}^{0}$ with the line $\varphi_{0}(k) \in \mathcal{E}_{2}^{0}$ to form a line of length $a_{k}$. In other words any interval $\left[0_{k}, \infty_{k}\right), k \in \mathcal{E}_{1}^{0}$ belonging to $\Gamma_{1}$ and the interval $\left[0_{\varphi_{0}(k)}, \infty_{\varphi_{0}(k)}\right)$ belonging to $\Gamma_{2}$ is replaced by the finite interval $\left[0_{k}, a_{k}\right]$ with $0_{k}$ being associated to the same vertex in $\Gamma_{1}$ as previously and $a_{k}$ being associated to the same vertex in $\Gamma_{2}$ as $0_{\varphi_{0}(k)}$ before in the sense of the discussion at the end of the previous section. Recall that the graphs need not be planar. Thus $\Gamma$ has $n=n_{1}+n_{2}-2 p$ external lines indexed by elements in $\left(\mathcal{E}_{1} \backslash \mathcal{E}_{1}^{0}\right) \cup\left(\mathcal{E}_{2} \backslash \mathcal{E}_{2}^{0}\right)$ and $p$ internal lines indexed by elements in $\varepsilon_{1}^{0}$ in addition to those of $\Gamma_{1}$ and of $\Gamma_{2}$. We denote this set by $\mathcal{J}_{12}$ such that the set of all internal lines of the graph $\Gamma$ is given by $\mathcal{J}=\mathcal{J}_{1} \cup \mathcal{J}_{2} \cup \mathcal{J}_{12}$. There are no new vertices in addition to those of $\Gamma_{1}$ and $\Gamma_{2}$ so the boundary conditions on $\Gamma_{1}$ and $\Gamma_{2}$ define boundary conditions on $\Gamma$ resulting in a Laplace operator $\Delta(\Gamma)$. Suppose that the indices of $\varepsilon_{1}^{0}$ in $\mathcal{E}_{1}$ come after the indices in $\mathcal{E}_{1} \backslash \mathcal{E}_{1}^{0}$ (in an arbitrary but fixed order) (see (3.1)). Via the map $\varphi_{0}$ we may identify $\varepsilon_{2}^{0}$ with $\varepsilon_{1}^{0}$ so let these indices now come first in $\varepsilon_{2}$, but again in the same order. Finally, let the diagonal $n_{2} \times n_{2}$ matrix $V(\underline{a})$ be given as

$$
V(\underline{a})=\left(\begin{array}{cc}
\exp i \sqrt{\lambda} \underline{a} & 0 \\
0 & \mathbb{I}
\end{array}\right),
$$

where $\exp (i \sqrt{\lambda} \underline{a})$ again is the diagonal $p \times p$ matrix given by the $p$ new lengths $a_{k}, k \in \mathcal{E}_{2}^{0}$. 
Recall that by Theorem 2.1 all eigenfunctions of the operator $-\Delta(\Gamma)$ have the form

$$
\psi= \begin{cases}0, & j \in \mathcal{E}, \\ \alpha_{j} e^{i \sqrt{\lambda} x}+\beta_{j} e^{-i \sqrt{\lambda} x}, & j \in \mathcal{J},\end{cases}
$$

where the coefficients $\alpha_{j}$ and $\beta_{j}$ satisfy the homogeneous equation

$$
Z_{A, B, \underline{a}}(\lambda)\left(\begin{array}{c}
0 \\
\alpha \\
\beta
\end{array}\right)=0, \quad \alpha=\left\{\alpha_{j}\right\}_{j=1}^{m}, \quad \beta=\left\{\beta_{j}\right\}_{j=1}^{m}
$$

with the matrix $Z_{A, B, \underline{a}}(\lambda)$ defined by (2.6). We define the linear subspace $\mathcal{L}_{12}(\lambda)$ of $\mathbb{C}^{n+2 m}$ as a set of all vectors $(0, \alpha, \beta)^{T}$ for which $\alpha_{j}=\beta_{j}=0$ for all $j \in \mathcal{J}_{12}$,

$$
\mathcal{L}_{12}(\lambda)=\left\{\ell=(0, \alpha, \beta)^{T} \in \operatorname{Ker} Z_{A, B, \underline{a}}(\lambda) \subset \mathbb{C}^{n+2 m} \mid \alpha_{j}=\beta_{j} \forall j \in \mathcal{J}_{12}\right\} .
$$

Obviously for $\lambda \notin \sigma_{A, B, \underline{a}}$ this subspace is trivial, i.e. $\mathcal{L}_{12}(\lambda)=\{0\}$. Let $\Upsilon\left(\Gamma, \mathcal{J}_{12}\right) \subset \mathbb{R}$ be the set of those eigenvalues of $-\Delta(\Gamma)$ for which $\operatorname{Ker} Z_{A, B, \underline{a}}(\lambda) \ominus \mathcal{L}_{12}(\lambda)$ is nontrivial. Obviously the eigenfunctions corresponding to the eigenvalues from $\Upsilon\left(\Gamma, \mathcal{J}_{12}\right)$ have nontrivial overlap with $\mathcal{J}_{12}$, i.e. supp $\phi \cap J_{12}$ has non-zero measure.

Let $\Xi\left(\Gamma_{1}, \Gamma_{2}\right) \subset \mathbb{R}_{+}$be the set of those $\lambda>0$ for which $\operatorname{Ker}\left(V(\underline{a}) S_{22}^{(1)}(\lambda) V(\underline{a}) S_{11}^{(2)}(\lambda)-1\right)$ is nontrivial.

Theorem 4.1. With the above notations $\Xi\left(\Gamma_{1}, \Gamma_{2}\right)=\Upsilon\left(\Gamma, \mathcal{J}_{12}\right) \cap \mathbb{R}_{+}$. The composition rule

$$
S(\lambda)=S_{1}(\lambda) *_{p} V(\underline{a}) S_{2}(\lambda) V(\underline{a})
$$

holds for all $\lambda \in \mathbb{R}_{+}$. If $\lambda \in \Upsilon\left(\Gamma, J_{12}\right)$ and $\lambda>0$ then its multiplicity equals

$$
\begin{gathered}
\operatorname{dim} \operatorname{Ker}\left(-\Delta\left(\Gamma_{1}\right)-\lambda\right)+\operatorname{dim} \operatorname{Ker}\left(-\Delta\left(\Gamma_{2}\right)-\lambda\right) \\
+\operatorname{dim} \operatorname{Ker}\left(V(\underline{a}) S_{22}^{(1)}(\lambda) V(\underline{a}) S_{11}^{(2)}(\lambda)-1\right) .
\end{gathered}
$$

In particular if the eigenvalue $\lambda>0$ is such that $\lambda \notin \Upsilon\left(\Gamma, J_{12}\right)$ then its multiplicity equals

$$
\operatorname{dim} \operatorname{Ker}\left(-\Delta\left(\Gamma_{1}\right)-\lambda\right)+\operatorname{dim} \operatorname{Ker}\left(-\Delta\left(\Gamma_{2}\right)-\lambda\right)
$$

Here $\operatorname{dim} \operatorname{Ker}\left(-\Delta\left(\Gamma_{l}\right)-\lambda\right)$ denotes the multiplicity of the eigenvalue $\lambda$ regardless whether it is embedded into the absolutely continuous spectrum or not.

Note that by Lemma $3.2 \operatorname{dim} \operatorname{Ker}\left(V(\underline{a}) S_{22}^{(1)}(\lambda) V(\underline{a}) S_{11}^{(2)}(\lambda)-1\right)$ equals the algebraic multiplicity of the eigenvalue $\mu=1$ of $V(\underline{a}) S_{22}^{(1)}(\lambda) V(\underline{a}) S_{11}^{(2)}(\lambda)$.

If by cutting $p$ internal lines of an arbitrary graph $\Gamma$ with local boundary conditions, the graph will be decomposed into two disjoint subgraphs $\Gamma_{1}$ and $\Gamma_{2}$, by Theorem 4.1 the scattering matrix $S_{\Gamma}$ can be obtained from the scattering matrices $S_{\Gamma_{1}}$ and $S_{\Gamma_{2}}$ at the same energy. Thus, using (4.2) iteratively the scattering matrix associated to any graph can be obtained from the scattering matrices associated to its subgraphs each having one vertex only. In fact, pick one vertex and choose all the internal lines connecting to all other vertices. This leads to two graphs and the rule (4.2) may be applied. Iterating this procedure $L$ times, where $L$ is the number of vertices, gives the desired result.

Proof of Theorem 4.1. We split the proof into several steps.

1. First we suppose that $S_{1}(\lambda)$ is compatible with $V(\underline{a}) S_{2}(\lambda) V(\underline{a})$ and prove that the composition rule (4.2) holds. Let $\psi_{j}^{k}\left(x, \lambda ; \Gamma_{l}\right), j \in \mathcal{E}_{l} \cup \mathcal{J}_{l}$ for any $k \in \mathcal{E}_{l}$ be the solution of the Schrödinger equation with the operator $-\Delta\left(\Gamma_{l}\right), l=1,2$ (see (2.4) ) at energy $\lambda$. Let $\Psi_{l}(x, \lambda)$ be $n_{l} \times n_{l}$ matrices

$$
\left[\Psi_{l}(x, \lambda)\right]_{j k}=\psi_{j}^{k}\left(x, \lambda ; \Gamma_{l}\right), \quad j, k \in \mathcal{E}_{l}, \quad l=1,2
$$

such that

$$
\Psi_{l}(x, \lambda)=e^{-i \sqrt{\lambda} x} \mathbb{I}+e^{i \sqrt{\lambda} x} S_{l}(\lambda), \quad l=1,2 .
$$



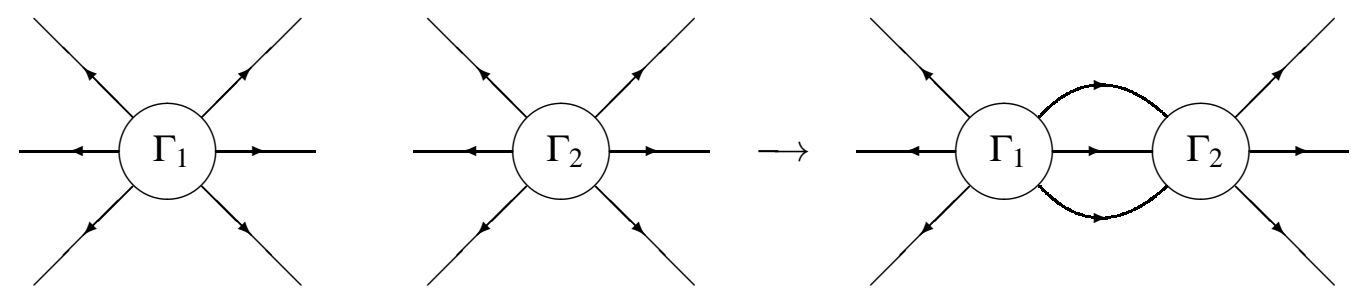

FIG. 1. Gluing of two graphs.

Observe that $e^{-i \sqrt{\lambda} x}$ and $e^{i \sqrt{\lambda} x}$ are linearly independent functions and therefore the scattering matrices may uniquely be recovered from $\Psi_{l}(x, \lambda)$. The columns of $\Psi_{l}(x, \lambda)$ define the external part of solutions of the Schrödinger equation for $-\Delta\left(\Gamma_{l}\right)$ at energy $\lambda$. We are looking for a square matrix

$$
\Psi(x, \lambda)=e^{-i \sqrt{\lambda} x} \mathbb{I}+e^{i \sqrt{\lambda} x} S(\lambda)
$$

such that its $\left(n_{1}-p\right)+\left(n_{2}-p\right)=n_{1}+n_{2}-2 p$ columns defines the external parts of a solution to the Schrödinger equation for $-\Delta(\Gamma)$. Here the indices are assumed to be arranged such that the first indices are those of $\mathcal{E}_{1} \backslash \mathcal{E}_{1}^{0}$ followed by the indices of $\varepsilon_{2} \backslash \varepsilon_{2}^{0}$. The aim is to obtain $\Psi(x, \lambda)$ from $\Psi_{1}(x, \lambda), \Psi_{2}(x, \lambda)$, and the lengths $\underline{a}=\left\{a_{s}\right\}_{s \in \mathcal{J}_{12}}$ of the new internal lines $\mathcal{J}_{12}$. By the above observation this will determine the scattering matrix $S(\lambda)$. The strategy will be to find new solutions of the Schrödinger equations for $-\Delta\left(\Gamma_{l}\right)$ with incoming plane waves $\left(e^{-i \sqrt{\lambda} x}\right)$ in the channels $k \in \mathcal{E}_{l} \backslash \mathcal{E}_{l}^{0}$ which agree suitably in the channels $k \in \mathcal{E}_{1}^{0}$ and $\varphi_{0}(k) \in \mathcal{E}_{2}^{0}$.

With the conventions made above we write

$$
\begin{aligned}
& S_{1}(\lambda)=\left(\begin{array}{cc}
S_{1}^{\left(n_{1}-p\right) \times\left(n_{1}-p\right)}(\lambda) & S_{1}^{\left(n_{1}-p\right) \times p}(\lambda) \\
S_{1}^{p \times\left(n_{1}-p\right)}(\lambda) & S_{1}^{p \times p}(\lambda)
\end{array}\right), \\
& S_{2}(\lambda)=\left(\begin{array}{cc}
S_{2}^{p \times p}(\lambda) & S_{2}^{p \times\left(n_{2}-p\right)}(\lambda) \\
S_{2}^{\left(n_{2}-p\right) \times p}(\lambda) & S_{2}^{\left(n_{2}-p\right) \times\left(n_{2}-p\right)}(\lambda)
\end{array}\right),
\end{aligned}
$$

where the superscripts denote the sizes of the blocks. For arbitrary $p \times\left(n_{1}-p\right)$ matrices $C_{1}$ and $C_{2}$, respectively, consider the $n_{1} \times\left(n_{1}-p\right)$ and $n_{2} \times\left(n_{1}-p\right)$ matrices

$$
\begin{aligned}
& \Phi_{1}\left(x, \lambda ; C_{1}\right)=e^{-i \sqrt{\lambda} x}\left(\begin{array}{c}
\mathbb{I} \\
C_{1}
\end{array}\right)+e^{i \sqrt{\lambda} x} S_{1}(\lambda)\left(\begin{array}{c}
\mathbb{I} \\
C_{1}
\end{array}\right), \\
& \Phi_{2}\left(x, \lambda ; C_{2}\right)=e^{-i \sqrt{\lambda} x}\left(\begin{array}{c}
C_{2} \\
0
\end{array}\right)+e^{i \sqrt{\lambda} x} S_{2}(\lambda)\left(\begin{array}{c}
C_{2} \\
0
\end{array}\right) .
\end{aligned}
$$

Here $\mathbb{I}$ stands for the $\left(n_{1}-p\right) \times\left(n_{1}-p\right)$ unit matrix and 0 stands for the $\left(n_{2}-p\right) \times\left(n_{1}-p\right)$ zero matrix. Obviously, the columns of $\Phi_{l}\left(x, \lambda ; C_{l}\right)$ are the external parts of linear combinations of the columns of $\Psi_{l}\left(x, \lambda ; \Gamma_{l}\right)$, and thus define the external parts of solutions of the Schrödinger equations for the operators $-\Delta\left(\Gamma_{l}\right), l=1,2$. Note that $\Phi_{1}\left(x, \lambda ; C_{1}\right)$ has an incoming plane wave in any of the channels $k \in \mathcal{E}_{1} \backslash \mathcal{E}_{1}^{0}$ and $\Phi_{2}\left(x, \lambda ; C_{2}\right)$ has no incoming plane wave in all channels $k \in \mathcal{E}_{2} \backslash \mathcal{E}_{2}^{0}$. 
Now we make the coordinate transformations $x \rightarrow a_{k}-x$ on the lines $\varphi_{0}(k) \in \mathcal{E}_{2}^{0}\left(k \in \mathcal{E}_{1}^{0}\right)$. The reason for this is as follows. Under the gluing process $\Gamma_{1}, \Gamma_{2} \rightarrow \Gamma$ (see Fig. 1) the two half-lines corresponding to $k \in \mathcal{E}_{1}^{0}$ and $\varphi_{0}(k) \in \mathcal{E}_{2}^{0}$ are replaced by the interval [0, $\left.a_{k}\right]$, giving the new lines in $\mathcal{J}_{12}$. This may be realized by identifying a point $P$ on the half-line corresponding to $k \in \mathcal{E}_{1}^{0}$ and with coordinate $x\left(0 \leq x \leq a_{k}\right)$ with the point $Q$ on the half-line corresponding to $\varphi_{0}(k) \in \mathcal{E}_{2}^{0}$ with coordinate $a_{k}-x$. In particular $x=a_{k}$ on the $k$-line corresponds to $x=0$ on the $\varphi_{0}(k)$-line and vice versa. Applying this transformation to $\Phi_{2}\left(x, \lambda ; C_{2}\right)$ we obtain in this new coordinate system

$$
\Phi_{2}^{(\underline{a})}\left(x, \lambda ; C_{2}\right)=\left(\begin{array}{cc}
e^{-i \sqrt{\lambda}(\underline{a}-x)} & 0 \\
0 & e^{-i \sqrt{\lambda} x}
\end{array}\right)\left(\begin{array}{c}
C_{2} \\
0
\end{array}\right)+\left(\begin{array}{cc}
e^{i \sqrt{\lambda}(\underline{a}-x)} & 0 \\
0 & e^{i \sqrt{\lambda} x}
\end{array}\right) S_{2}(\lambda)\left(\begin{array}{c}
C_{2} \\
0
\end{array}\right) .
$$

We now require that $\Phi_{1}\left(x, \lambda ; C_{1}\right)$ and $\Phi_{2}^{(a)}\left(x, \lambda ; C_{2}\right)$ agree on the lines labeled by $\mathcal{J}_{12}$. This will fix $C_{1}$ and $C_{2}$. Indeed, we then obtain

$$
\begin{aligned}
e^{-i \sqrt{\lambda} x} C_{1}+ & e^{i \sqrt{\lambda} x} S_{1}^{p \times\left(n_{1}-p\right)}(\lambda)+e^{i \sqrt{\lambda} x} S_{1}^{p \times p}(\lambda) C_{1} \\
& =e^{-i \sqrt{\lambda}(\underline{a}-x)} C_{2}+e^{i \sqrt{\lambda}(\underline{a}-x)} S_{2}^{p \times p}(\lambda) C_{2} .
\end{aligned}
$$

By the linear independence of the functions $e^{i \sqrt{\lambda} x}$ and $e^{-i \sqrt{\lambda} x}$ it follows that

$$
\begin{aligned}
& C_{1}=e^{i \sqrt{\lambda} \underline{a}} S_{2}^{p \times p}(\lambda) C_{2}, \\
& S_{1}^{p \times p}(\lambda) C_{1}+S_{1}^{p \times\left(n_{1}-p\right)}(\lambda)=e^{-i \sqrt{\lambda}} \underline{a} C_{2},
\end{aligned}
$$

and thus

$$
\begin{aligned}
& C_{2}=\left[\mathbb{I}-e^{i \sqrt{\lambda} \underline{a}} S_{1}^{p \times p}(\lambda) e^{i \sqrt{\lambda} \underline{a}} S_{2}^{p \times p}(\lambda)\right]^{-1} e^{i \sqrt{\lambda} \underline{a}} S_{1}^{p \times\left(n_{1}-p\right)}(\lambda), \\
& C_{1}=e^{i \sqrt{\lambda} \underline{a}} S_{2}^{p \times p}(\lambda)\left[\mathbb{I}-e^{i \sqrt{\lambda} \underline{a}} S_{1}^{p \times p}(\lambda) e^{i \sqrt{\lambda} \underline{a}} S_{2}^{p \times p}(\lambda)\right]^{-1} e^{i \sqrt{\lambda} \underline{a}} S_{1}^{p \times\left(n_{1}-p\right)}(\lambda) .
\end{aligned}
$$

Since for any invertible $A$ and $U$ the identities $U A^{-1}=\left(A U^{-1}\right)^{-1}$ and $A^{-1} U=\left(U^{-1} A\right)^{-1}$ hold, we have

$$
\begin{aligned}
& {\left[\mathbb{I}-e^{i \sqrt{\lambda} \underline{a}} S_{1}^{p \times p}(\lambda) e^{i \sqrt{\lambda} \underline{a}} S_{2}^{p \times p}(\lambda)\right]^{-1} e^{i \sqrt{\lambda} \underline{a}}} \\
& \quad=\left[e^{-i \sqrt{\lambda} \underline{a}}-S_{1}^{p \times p}(\lambda) e^{i \sqrt{\lambda} \underline{a}} S_{2}^{p \times p}(\lambda)\right]^{-1} \\
& =e^{i \sqrt{\lambda} \underline{a}}\left[\mathbb{I}-S_{1}^{p \times p}(\lambda) e^{i \sqrt{\lambda} \underline{a}} S_{2}^{p \times p}(\lambda) e^{i \sqrt{\lambda} \underline{a}}\right]^{-1} .
\end{aligned}
$$

Since $S_{1}(\lambda)$ is assumed to be compatible with $V(\underline{a}) S_{2}(\lambda) V(\underline{a})$ the inverses in (4.8) are well defined.

Similar to (4.5) and according to the ordering convention made above we write the scattering matrix $S(\lambda)$ for the graph $\Gamma$ in the block form

$$
S(\lambda)=\left(\begin{array}{ll}
S^{\left(n_{1}-p\right) \times\left(n_{1}-p\right)}(\lambda) & S^{\left(n_{1}-p\right) \times\left(n_{2}-p\right)}(\lambda) \\
S^{\left(n_{2}-p\right) \times\left(n_{1}-p\right)}(\lambda) & S^{\left(n_{2}-p\right) \times\left(n_{2}-p\right)}(\lambda)
\end{array}\right),
$$

where the superscripts denote again the sizes of the blocks. Since $\Phi_{1}\left(x, \lambda ; C_{1}\right)$ has an incoming plane wave in any of the first $n_{1}-p$ channels $k \in \mathcal{E}_{1} \backslash \mathcal{E}_{1}^{0}$, equations (4.8) allow one to determine 
$S^{\left(n_{1}-p\right) \times\left(n_{1}-p\right)}(\lambda)$ and $S^{\left(n_{1}-p\right) \times\left(n_{2}-p\right)}(\lambda)$ :

$$
\begin{aligned}
S^{\left(n_{1}-p\right) \times\left(n_{1}-p\right)}(\lambda)= & S_{1}^{\left(n_{1}-p\right) \times\left(n_{1}-p\right)}(\lambda)+S_{1}^{\left(n_{1}-p\right) \times p}(\lambda) C_{1} \\
= & S_{1}^{\left(n_{1}-p\right) \times\left(n_{1}-p\right)}(\lambda)+S_{1}^{\left(n_{1}-p\right) \times p}(\lambda) e^{i \sqrt{\lambda} \underline{a}} S_{2}^{p \times p}(\lambda) e^{i \sqrt{\lambda} \underline{a}} \\
& \cdot\left[\mathbb{I}-S_{1}^{p \times p}(\lambda) e^{i \sqrt{\lambda} \underline{a}} S_{2}^{p \times p}(\lambda) e^{i \sqrt{\lambda} \underline{a}}\right]^{-1} S_{1}^{p \times\left(n_{1}-p\right)}(\lambda), \\
S^{\left(n_{2}-p\right) \times\left(n_{1}-p\right)}(\lambda)= & S_{2}^{\left(n_{2}-p\right) \times p}(\lambda) C_{2} \\
= & S_{2}^{\left(n_{2}-p\right) \times p}(\lambda) e^{i \sqrt{\lambda} \underline{a}} \\
& \cdot\left[\mathbb{I}-S_{1}^{p \times p}(\lambda) e^{i \sqrt{\lambda} \underline{a}} S_{2}^{p \times p}(\lambda) e^{i \sqrt{\lambda} \underline{a}}\right]^{-1} S_{1}^{p \times\left(n_{1}-p\right)}(\lambda) .
\end{aligned}
$$

To determine the remaining blocks of the scattering matrix $S(\lambda)$ instead of (4.6) we consider the $n_{1} \times\left(n_{2}-p\right)$ and $n_{2} \times\left(n_{2}-p\right)$ matrices

$$
\begin{aligned}
& \widetilde{\Phi}_{1}\left(x, \lambda ; \widetilde{C}_{1}\right)=e^{-i \sqrt{\lambda} x}\left(\begin{array}{c}
0 \\
\widetilde{C}_{1}
\end{array}\right)+e^{i \sqrt{\lambda} x} S_{1}(\lambda)\left(\begin{array}{c}
0 \\
\widetilde{C}_{1}
\end{array}\right), \\
& \widetilde{\Phi}_{2}\left(x, \lambda ; \widetilde{C}_{2}\right)=e^{-i \sqrt{\lambda} x}\left(\begin{array}{c}
\widetilde{C}_{2} \\
\mathbb{I}
\end{array}\right)+e^{i \sqrt{\lambda} x} S_{2}(\lambda)\left(\begin{array}{c}
\widetilde{C}_{2} \\
\mathbb{I}
\end{array}\right) .
\end{aligned}
$$

with arbitrary $p \times\left(n_{2}-p\right)$ matrices $\widetilde{C}_{1}$ and $\widetilde{C}_{2}$. Again $\widetilde{\Phi}_{l}\left(x, \lambda ; \widetilde{C}_{l}\right)$ are the external parts of some solutions of the Schrödinger equations with the operators $-\Delta\left(\Gamma_{l}\right), l=1,2$. Now $\widetilde{\Phi}_{1}\left(x, \lambda ; \widetilde{C}_{1}\right)$ has no incoming plane waves in the channels $k \in \mathcal{E}_{1} \backslash \mathcal{E}_{1}^{0}$, but $\widetilde{\Phi}_{2}\left(x, \lambda ; \widetilde{C}_{2}\right)$ has an incoming plane wave in any of the channels $k \in \mathcal{E}_{2} \backslash \mathcal{E}_{2}^{0}$. $\widetilde{C}_{2}$ :

Repeating the arguments used above we obtain the following matching conditions for $\widetilde{C}_{1}$ and

$$
\begin{aligned}
& \widetilde{C}_{2}=e^{i \sqrt{\lambda} \underline{a}} S_{1}^{p \times p} \widetilde{C}_{1}, \\
& S_{2}^{p \times p}(\lambda) \widetilde{C}_{2}+S_{2}^{p \times\left(n_{2}-p\right)}(\lambda)=e^{-i \sqrt{\lambda} \underline{a}} \widetilde{C}_{1},
\end{aligned}
$$

and thus

$$
\begin{aligned}
& \widetilde{C}_{1}=\left[\mathbb{I}-e^{i \sqrt{\lambda} \underline{a}} S_{2}^{p \times p}(\lambda) e^{i \sqrt{\lambda} \underline{a}} S_{1}^{p \times p}(\lambda)\right]^{-1} e^{i \sqrt{\lambda} \underline{a}} S_{2}^{p \times\left(n_{2}-p\right)}(\lambda), \\
& \widetilde{C}_{2}=e^{i \sqrt{\lambda} \underline{a}} S_{1}^{p \times p}(\lambda)\left[\mathbb{I}-e^{i \sqrt{\lambda} \underline{a}} S_{2}^{p \times p}(\lambda) e^{i \sqrt{\lambda} \underline{a}} S_{1}^{p \times p}(\lambda)\right]^{-1} e^{i \sqrt{\lambda} \underline{a}} S_{2}^{p \times\left(n_{2}-p\right)}(\lambda) .
\end{aligned}
$$

Since $S_{1}(\lambda)$ is compatible with $V(\underline{a}) S_{2}(\lambda) V(\underline{a})$ the inverses are again well defined. From this and from (4.9) it follows that

$$
\begin{aligned}
S^{\left(n_{2}-p\right) \times\left(n_{2}-p\right)}(\lambda)= & S_{2}^{\left(n_{2}-p\right) \times\left(n_{2}-p\right)}(\lambda)+S_{2}^{\left(n_{2}-p\right) \times p}(\lambda) \widetilde{C}_{2} \\
= & S_{2}^{\left(n_{2}-p\right) \times\left(n_{2}-p\right)}(\lambda)+S_{2}^{\left(n_{2}-p\right) \times p}(\lambda) e^{i \sqrt{\lambda} \underline{a}} S_{1}^{p \times p}(\lambda) \\
& \quad \cdot\left[\mathbb{I}-e^{i \sqrt{\lambda} \underline{a}} S_{2}^{p \times p}(\lambda) e^{i \sqrt{\lambda}} \underline{a} S_{1}^{p \times p}(\lambda)\right]^{-1} e^{i \sqrt{\lambda} \underline{a}} S_{2}^{p \times\left(n_{2}-p\right)}(\lambda), \\
S^{\left(n_{1}-p\right) \times\left(n_{2}-p\right)}(\lambda)= & S_{1}^{\left(n_{1}-p\right) \times p}(\lambda) \widetilde{C}_{1} \\
= & S_{1}^{\left(n_{1}-p\right) \times p}(\lambda) \\
& \quad \cdot\left[\mathbb{I}-e^{i \sqrt{\lambda} \underline{a}} S_{2}^{p \times p}(\lambda) e^{i \sqrt{\lambda}} \underline{a} S_{1}^{p \times p}(\lambda)\right]^{-1} e^{i \sqrt{\lambda} \underline{a}} S_{2}^{p \times\left(n_{2}-p\right)}(\lambda) .
\end{aligned}
$$

By the definition of the generalized star product (3.4) we obtain (4.2). 
2. Now suppose that $\lambda \in \Xi\left(\Gamma_{1}, \Gamma_{2}\right)$. We prove that the composition rule (4.2) remains valid. Also $\lambda \in \Upsilon\left(\Gamma, \mathcal{J}_{12}\right)$ and the multipicity of $\lambda$ equals

$$
\begin{gathered}
\operatorname{dim} \operatorname{Ker}\left(-\Delta\left(\Gamma_{1}\right)-\lambda\right)+\operatorname{dim} \operatorname{Ker}\left(-\Delta\left(\Gamma_{2}\right)-\lambda\right) \\
+\operatorname{dim} \operatorname{Ker}\left(V(\underline{a}) S_{22}^{(1)}(\lambda) V(\underline{a}) S_{11}^{(2)}(\lambda)-1\right) .
\end{gathered}
$$

The assumption $\lambda \in \Xi\left(\Gamma_{1}, \Gamma_{2}\right)$ implies that

$$
\mathbb{I}-S_{1}^{p \times p}(\lambda) e^{i \sqrt{\lambda} \underline{a}} S_{2}^{p \times p}(\lambda) e^{i \sqrt{\lambda} \underline{a}}
$$

and

$$
\mathbb{I}-e^{i \sqrt{\lambda} \underline{a}} S_{2}^{p \times p}(\lambda) e^{i \sqrt{\lambda} \underline{a}} S_{1}^{p \times p}(\lambda)
$$

have nontrivial kernels. This implies that the homogeneous form of the equations $(4.7)$ and (4.10),

$$
\begin{aligned}
& C_{1}=e^{i \sqrt{\lambda} \underline{a}} S_{2}^{p \times p}(\lambda) C_{2}, \\
& S_{1}^{p \times p}(\lambda) C_{1}=e^{-i \sqrt{\lambda} \underline{a}} C_{2},
\end{aligned}
$$

and

$$
\begin{aligned}
& \widetilde{C}_{2}=e^{i \sqrt{\lambda} \underline{a}} S_{1}^{p \times p}(\lambda) \widetilde{C}_{1}, \\
& S_{2}^{p \times p}(\lambda) \widetilde{C}_{2}=e^{-i \sqrt{\lambda} \underline{a}} \widetilde{C}_{1},
\end{aligned}
$$

respectively, have nontrivial solutions. It is easy to prove that the inhomogeneous equations (4.7) and (4.10) still have solutions in this case. Consider for instance the equation (4.7), which is equivalent to

$$
C_{2}=e^{i \sqrt{\lambda} \underline{a}} S_{1}^{p \times\left(n_{1}-p\right)}(\lambda)+e^{i \sqrt{\lambda} \underline{a}} S_{1}^{p \times p}(\lambda) e^{i \sqrt{\lambda} \underline{a}} S_{2}^{p \times p}(\lambda) C_{2} .
$$

By the Fredholm alternative this equation has a non-trivial solution iff

$$
S_{1}^{p \times\left(n_{1}-p\right)}(\lambda)^{*} e^{-i \sqrt{\lambda} \underline{a}} b=0
$$

for any $0 \neq b \in \mathbb{C}^{p}$ satisfying

$$
S_{2}^{p \times p}(\lambda)^{*} e^{-i \sqrt{\lambda} \underline{a}} S_{1}^{p \times p}(\lambda)^{*} e^{-i \sqrt{\lambda} \underline{a}} b=b .
$$

By Lemma 3.3 with $A=S_{2}^{p \times p}(\lambda)^{*}$ and $B=e^{-i \sqrt{\lambda} \underline{a}} S_{1}^{p \times p}(\lambda)^{*} e^{-i \sqrt{\lambda} \underline{a}}$ we have

$$
e^{i \sqrt{\lambda} \underline{a}} S_{1}^{p \times p}(\lambda) S_{1}^{p \times p}(\lambda)^{*} e^{-i \sqrt{\lambda} \underline{a}} b=b .
$$

From the unitarity of $V(\underline{a}) S_{1}(\lambda) V(\underline{a})$, which states in particular that

$$
\begin{aligned}
& e^{i \sqrt{\lambda} \underline{a}} S_{1}^{p \times p}(\lambda) S_{1}^{p \times p}(\lambda)^{*} e^{-i \sqrt{\lambda} \underline{a}} \\
& \quad+e^{i \sqrt{\lambda} \underline{a}} S_{1}^{p \times\left(n_{1}-p\right)}(\lambda) S_{1}^{p \times\left(n_{1}-p\right)}(\lambda)^{*} e^{-i \sqrt{\lambda} \underline{a}}=\mathbb{I},
\end{aligned}
$$

and from (4.15) it follows that

$$
e^{i \sqrt{\lambda} \underline{a}} S_{1}^{p \times\left(n_{1}-p\right)}(\lambda) S_{1}^{p \times\left(n_{1}-p\right)}(\lambda)^{*} e^{-i \sqrt{\lambda} \underline{a}} b=0 .
$$

Since $\operatorname{Ker} C^{*} C=\operatorname{Ker} C$ for any operator $C$ we obtain 4.14 . Equation 4.10 ) is discussed similarly.

From (4.6) and (4.9) it follows that the Schrödinger equation with the operator $-\Delta(\Gamma)$ for given value of the spectral parameter $\lambda>0$ has (nonunique) solutions which have the form

$$
\begin{gathered}
\Phi_{1}\left(x, \lambda ; C_{1}\right)=e^{-i \sqrt{\lambda} x}\left(\begin{array}{c}
0 \\
C_{1}
\end{array}\right)+e^{i \sqrt{\lambda} x} S_{1}(\lambda)\left(\begin{array}{c}
0 \\
C_{1}
\end{array}\right), \\
\Phi_{2}\left(x, \lambda ; C_{2}\right)=e^{-i \sqrt{\lambda} x}\left(\begin{array}{c}
C_{2} \\
0
\end{array}\right)+e^{i \sqrt{\lambda} x} S_{2}(\lambda)\left(\begin{array}{c}
C_{2} \\
0
\end{array}\right)
\end{gathered}
$$


and

$$
\begin{aligned}
& \widetilde{\Phi}_{1}\left(x, \lambda ; \widetilde{C}_{1}\right)=e^{-i \sqrt{\lambda} x}\left(\begin{array}{c}
0 \\
\widetilde{C}_{1}
\end{array}\right)+e^{i \sqrt{\lambda} x} S_{1}(\lambda)\left(\begin{array}{c}
0 \\
\widetilde{C}_{1}
\end{array}\right), \\
& \widetilde{\Phi}_{2}\left(x, \lambda ; \widetilde{C}_{2}\right)=e^{-i \sqrt{\lambda} x}\left(\begin{array}{c}
\widetilde{C}_{2} \\
0
\end{array}\right)+e^{i \sqrt{\lambda} x} S_{2}(\lambda)\left(\begin{array}{c}
\widetilde{C}_{2} \\
0
\end{array}\right),
\end{aligned}
$$

where $C_{1}$ and $C_{2}\left(\widetilde{C}_{1}\right.$ and $\left.\widetilde{C}_{2}\right)$ solve (4.12) ((4.13), respectively). Note that $C_{1}=\widetilde{C}_{1}$ and $C_{2}=\widetilde{C}_{2}$. On the lines in the set $J_{12}$ the quantity $\Phi_{1}$ coincides with $\Phi_{2}$ and $\widetilde{\Phi}_{1}$ and $\widetilde{\Phi}_{2}$. We will now prove that

$$
S_{1}^{\left(n_{1}-p\right) \times p}(\lambda) C_{1}=0, \quad S_{2}^{\left(n_{2}-p\right) \times p}(\lambda) C_{2}=0 .
$$

Thus, the functions (4.16) and (4.17) are zero on all external lines of the graph $\Gamma$ and their support has nontrivial overlap with the interval lines $J_{12}$.

From (4.12) it follows that $C_{1}=e^{i \sqrt{\lambda} \underline{a}} S_{2}^{p \times p}(\lambda) e^{i \sqrt{\lambda} \underline{a}} S_{1}^{p \times p}(\lambda) C_{1}$. By Lemma 3.3 we have

$$
S_{1}^{p \times p}(\lambda)^{*} S_{1}^{p \times p}(\lambda) C_{1}=C_{1} .
$$

By unitarity it follows that

$$
S_{1}^{\left(n_{1}-p\right) \times p}(\lambda)^{*} S_{1}^{\left(n_{1}-p\right) \times p}(\lambda) C_{1}=0
$$

and thus $S_{1}^{\left(n_{1}-p\right) \times p}(\lambda) C_{1}=0$. The second relation in 4.18$)$ is proved similarly.

Now we note that from (4.12) and (4.13) it follows that

$$
\operatorname{Rank} C_{1}=\operatorname{Rank} C_{2}=\operatorname{dim} \operatorname{Ker}\left(e^{i \sqrt{\lambda} \underline{a}} S_{2}^{p \times p}(\lambda) e^{i \sqrt{\lambda} \underline{a}} S_{1}^{p \times p}(\lambda)-1\right) .
$$

The columns of (4.16) correspond to linear independent eigenfunctions of $-\Delta(\Gamma)$ for the eigenvalue $\lambda$. There are precisely $\operatorname{dim} \operatorname{Ker}\left(e^{i \sqrt{\lambda} \underline{a}} S_{2}^{p \times p}(\lambda) e^{i \sqrt{\lambda}} \underline{\underline{a}} S_{1}^{p \times p}(\lambda)-1\right)$ such eigenfunctions and the supports of all them have nontrivial overlap with the internal lines $\mathcal{J}_{12}$.

3. Let $\lambda \in \Upsilon\left(\Gamma, \mathcal{J}_{12}\right) \cap \mathbb{R}_{+}$and let

$$
\operatorname{dim}\left(\operatorname{Ker} Z_{A, B, \underline{a}}(\lambda) \ominus \mathcal{L}_{12}(\lambda)\right)=k,
$$

where the linear subspace $\mathcal{L}_{12}(\lambda)$ is defined by $(4.1)$. This means that there are precisely $k$ eigenfunctions of $-\Delta(\Gamma)$ which disappear if we cut the internal lines $J_{12}$. We will prove that $\lambda \in \Xi\left(\Gamma_{1}, \Gamma_{2}\right)$ and that

$$
\operatorname{dim} \operatorname{Ker}\left(V(\underline{a}) S_{22}^{(1)}(\lambda) V(\underline{a}) S_{11}^{(2)}(\lambda)-1\right)=k
$$

which in turn implies that

$$
\begin{array}{r}
\operatorname{dim} \operatorname{Ker}(-\Delta(\Gamma)-\lambda)=\operatorname{dim} \operatorname{Ker}\left(-\Delta\left(\Gamma_{1}\right)-\lambda\right)+\operatorname{dim} \operatorname{Ker}\left(-\Delta\left(\Gamma_{2}\right)-\lambda\right) \\
+\operatorname{dim} \operatorname{Ker}\left(V(\underline{a}) S_{22}^{(1)}(\lambda) V(\underline{a}) S_{11}^{(2)}(\lambda)-1\right) .
\end{array}
$$

From the existence of the above mentioned eigenfunctions it follows that these eigenfunctions can be constructed by means of superposition and matching of the solutions (4.4) of the Schrödinger equation for the operators $-\Delta\left(\Gamma_{1}\right)$ and $-\Delta\left(\Gamma_{2}\right)$ at energy $\lambda>0$. For any vectors $C_{1}, C_{2} \in \mathbb{C}^{p}$ the functions

$$
\begin{array}{r}
\phi_{1}\left(x, \lambda, C_{1}\right)=e^{-i \sqrt{\lambda} x}\left(\begin{array}{c}
0 \\
C_{1}
\end{array}\right)+e^{i \sqrt{\lambda} x} S_{1}(\lambda)\left(\begin{array}{c}
0 \\
C_{1}
\end{array}\right), \\
\phi_{2}\left(x, \lambda, C_{2}\right)=e^{-i \sqrt{\lambda} x}\left(\begin{array}{c}
C_{2} \\
0
\end{array}\right)+e^{i \sqrt{\lambda} x} S_{2}(\lambda)\left(\begin{array}{c}
C_{2} \\
0
\end{array}\right)
\end{array}
$$


define the external parts of solutions of the Schrödinger equations for the operators $-\Delta\left(\Gamma_{l}\right)$, $l=1,2$. Since the eigenfunctions are supported on internal lines of the graph $\Gamma$ (Theorem 2.1) the vectors $C_{1}$ and $C_{2}$ must satisfy

$$
S_{1}^{\left(n_{1}-p\right) \times p}(\lambda) C_{1}=0, \quad S_{2}^{p \times\left(n_{2}-p\right)}(\lambda) C_{2}=0
$$

such that $\phi_{1}\left(x, \lambda, C_{1}\right)$ vanishes in any of the channels $k \in \mathcal{E}_{1} \backslash \mathcal{E}_{1}^{0}$ and $\phi_{2}\left(x, \lambda, C_{2}\right)$ vanishes in all channels $k \in \mathcal{E}_{2} \backslash \mathcal{E}_{2}^{0}$. Making the coordinate transformation $x \rightarrow \underline{a}-x$ on the lines $\varphi_{0}(k) \in \mathcal{E}_{2}^{0}\left(k \in \mathcal{E}_{1}^{0}\right)$ and requiring that $\phi_{1}\left(x, \lambda, C_{1}\right)$ and $\phi_{2}\left(\underline{a}-x, \lambda, C_{2}\right)$ agree on the lines labeled by $\mathcal{J}_{12}$, we obtain

$$
\begin{gathered}
C_{1}=e^{i \sqrt{\lambda} \underline{a}} S_{2}^{p \times p}(\lambda) C_{2}, \\
S_{1}^{p \times p}(\lambda) C_{1}=e^{-i \sqrt{\lambda}} \underline{a} C_{2},
\end{gathered}
$$

or equivalently

$$
\begin{aligned}
& e^{i \sqrt{\lambda} \underline{a}} S_{2}^{p \times p}(\lambda) e^{i \sqrt{\lambda} \underline{a}} S_{1}^{p \times p}(\lambda) C_{1}=C_{1}, \\
& C_{2}=e^{i \sqrt{\lambda} \underline{a}} S_{1}^{p \times p}(\lambda) C_{1} .
\end{aligned}
$$

Linear independent solutions of (4.21) correspond to linear independent eigenfunctions of $-\Delta(\Gamma)$ and vise versa. Thus the condition (4.19) implies (4.20). This completes the proof of the theorem.

Note that if $\Gamma$ is simply the disjoint union of $\Gamma_{1}$ and $\Gamma_{2}$, i.e. if no connections are made (corresponding to $p=0$ and $\left.n=n_{1}+n_{2}\right)$, then $S(\lambda)$ is just the direct sum of $S_{1}(\lambda)$ and $S_{2}(\lambda)$. Also $V^{*} S(\lambda) V=S_{2 n}^{\text {free }}(\lambda) *_{V} S(\lambda)$ for any scattering matrix with $n$ open ends and any unitary $n \times n$ matrix $V$, where

$$
S_{2 n}^{\mathrm{free}}(\lambda)=\left(\begin{array}{ll}
0 & \mathbb{I} \\
\mathbb{I} & 0
\end{array}\right)
$$

Similarly $S(\lambda) *_{V} S_{2 n}^{\text {free }}(\lambda)=V S(\lambda) V^{*}$.

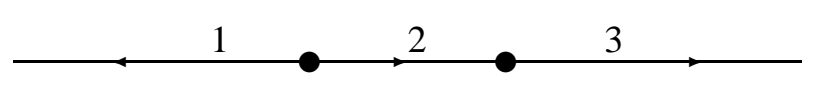

FIG. 2. The graph from Example 4.2.

Example 4.2. Consider an arbitrary self-adjoint Laplacian $\Delta(A, B)$ with local boundary conditions on the graph depicted in Fig. 2, where the distance between the two vertices is $a$. The composition rule (4.2) with

$$
V(a)=\left(\begin{array}{cc}
e^{i \sqrt{\lambda} a} & 0 \\
0 & 1
\end{array}\right)
$$


easily gives

$$
\begin{aligned}
& S_{11}=S_{11}^{(1)}+S_{12}^{(1)} S_{11}^{(2)} S_{21}^{(1)}\left(1-S_{22}^{(1)} S_{11}^{(2)} e^{2 i a \sqrt{\lambda}}\right)^{-1}, \\
& S_{22}=S_{22}^{(2)}+S_{22}^{(1)} S_{21}^{(2)} S_{12}^{(2)}\left(1-S_{22}^{(1)} S_{11}^{(2)} e^{2 i a \sqrt{\lambda}}\right)^{-1}, \\
& S_{12}=S_{12}^{(1)} S_{12}^{(2)}\left(1-S_{22}^{(1)} S_{11}^{(2)} e^{2 i a \sqrt{\lambda}}\right)^{-1}, \\
& S_{21}=S_{21}^{(2)} S_{21}^{(1)}\left(1-S_{22}^{(1)} S_{11}^{(2)} e^{2 i a \sqrt{\lambda}}\right)^{-1},
\end{aligned}
$$

where the $S$-matrices are written in the form analogous to (3.1)

$$
S^{(1)}=\left(\begin{array}{ll}
S_{11}^{(1)} & S_{12}^{(1)} \\
S_{21}^{(1)} & S_{22}^{(1)}
\end{array}\right), \quad S^{(2)}=\left(\begin{array}{ll}
S_{11}^{(2)} & S_{12}^{(2)} \\
S_{21}^{(2)} & S_{22}^{(2)}
\end{array}\right),
$$

leaving out the $\lambda$-dependence. These relations are equivalent to the well-known factorization formula [57, 58, 47, 1, 62, 64, 65] applied to the Laplacian on a line with boundary conditions posed at $x=0$ and $x=a$.

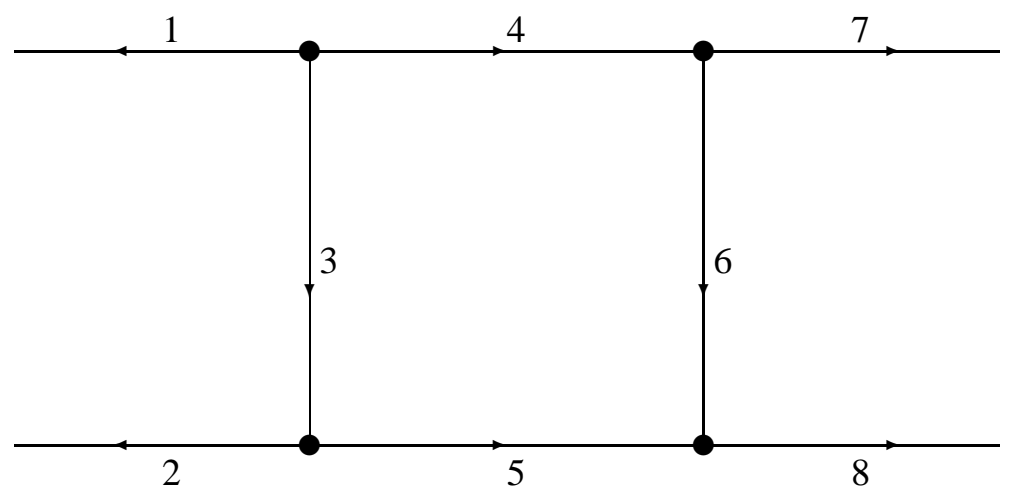

FIG. 3. The graph from Example 4.3. The arrows show the positive direction for every edge. The edges 3 and 6 have the length $a$ and the edges 4 and 5 the length $b$.

Example 4.3. Consider the graph depicted in Fig. 3 where the length of the edges 3 and 6 equals $a$ and the length of the edges 4 and 5 equals $b$. Let the boundary conditions be given as

$$
\begin{array}{r}
\psi_{1}(0)=\psi_{3}(0)=\psi_{4}(0), \\
\psi_{2}(0)=\psi_{3}(a)=\psi_{5}(0), \\
\psi_{1}^{\prime}(0)+\psi_{3}^{\prime}(0)+\psi_{4}^{\prime}(0)=0, \\
\psi_{2}^{\prime}(0)+\psi_{5}^{\prime}(0)-\psi_{3}^{\prime}(a)=0, \\
\psi_{4}(b)=\psi_{6}(0)=\psi_{7}(0), \\
\psi_{5}(b)=\psi_{6}(a)=\psi_{8}(0), \\
-\psi_{4}^{\prime}(b)+\psi_{6}^{\prime}(0)+\psi_{7}^{\prime}(0)=0, \\
-\psi_{5}^{\prime}(b)-\psi_{6}^{\prime}(a)+\psi_{8}^{\prime}(0)=0 .
\end{array}
$$

Obviously they define a self-adjoint operator which we denote by $\Delta(a, b)$. The scattering matrix corresponding to this operator (as defined by (2.3) and (2.4)) will be denoted by $S_{a, b}(\lambda)$. To determine this $4 \times 4$ matrix we first consider the graph depicted in Fig. $甘$ where the length of 
the edge 3 is supposed to be equal a. The boundary conditions (4.23) determine the self-adjoint operator. The corresponding scattering matrix we denote by $S_{a}(\lambda)$. From (2.7) it follows that

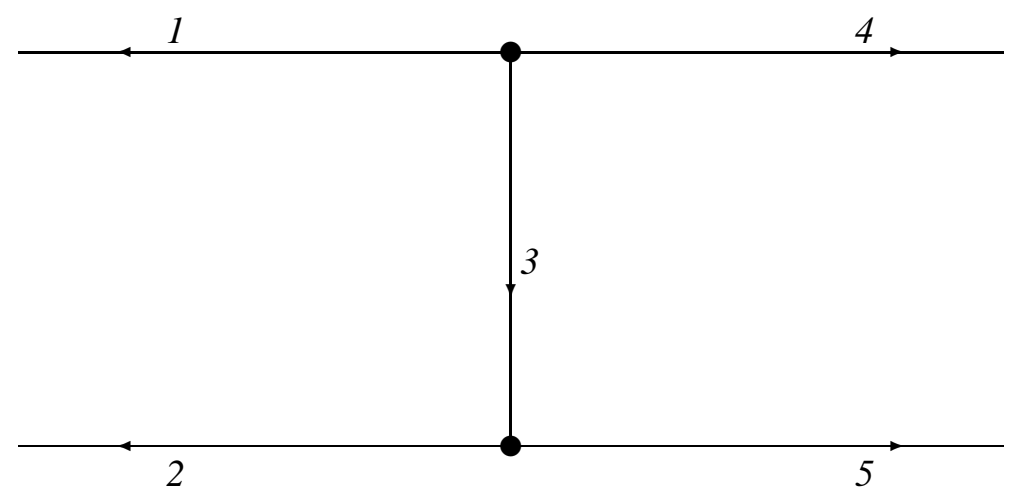

FIG. 4. The graph from Example 4.3. The arrows show the positive direction for every edge.

$$
\begin{gathered}
S_{a}(\lambda)=\left(e^{i \sqrt{\lambda} a}-9 e^{-i \sqrt{\lambda} a}\right)^{-1} \\
\cdot\left(\begin{array}{cccc}
e^{i \sqrt{\lambda} a}+3 e^{-i \sqrt{\lambda} a} & -4 & 2\left(e^{i \sqrt{\lambda} a}-3 e^{-i \sqrt{\lambda} a}\right) & -4 \\
-4 & e^{i \sqrt{\lambda} a}+3 e^{-i \sqrt{\lambda} a} & -4 & 2\left(e^{i \sqrt{\lambda} a}-3 e^{-i \sqrt{\lambda} a}\right) \\
2\left(e^{i \sqrt{\lambda} a}-3 e^{-i \sqrt{\lambda} a}\right) & -4 & e^{i \sqrt{\lambda} a}+3 e^{-i \sqrt{\lambda} a} & -4 \\
-4 & 2\left(e^{i \sqrt{\lambda} a}-3 e^{-i \sqrt{\lambda} a}\right) & -4 & e^{i \sqrt{\lambda} a}+3 e^{-i \sqrt{\lambda} a}
\end{array}\right) .
\end{gathered}
$$

By Theorem 4.1 the scattering matrix $S_{a, b}(\lambda)$ is given by

$$
S_{a, b}(\lambda)=S_{a}(\lambda) *_{2} V(\underline{b}) S_{a}(\lambda) V(\underline{b})
$$

where

$$
V(\underline{b})=\operatorname{diag}\left(e^{i \sqrt{\lambda} b}, e^{i \sqrt{\lambda} b}, 1,1\right), \quad \underline{b}=(b, b) \in \mathbb{R}^{2} .
$$

We now compute the $2 \times 2$ matrices $K_{1}$ and $K_{2}$ entering the definition (3.4) of the generalized star product, thus obtaining

$$
\begin{aligned}
& K_{1}^{-1}=K_{2}^{-1}=\left(e^{i \sqrt{\lambda} a}-9 e^{-i \sqrt{\lambda} a}\right)^{-2} L, \\
& (L)_{11}=(L)_{22}=e^{2 i \sqrt{\lambda} a}\left(1-e^{2 i \sqrt{\lambda} b}\right)+9 e^{-2 i \sqrt{\lambda} a}\left(9-e^{2 i \sqrt{\lambda} b}\right)-2\left(9+11 e^{2 i \sqrt{\lambda} b}\right), \\
& (L)_{12}=(L)_{21}=8 e^{2 i \sqrt{\lambda} b}\left(e^{i \sqrt{\lambda} a}+3 e^{-i \sqrt{\lambda} a}\right) .
\end{aligned}
$$

From this it follows that

$$
\begin{aligned}
& \operatorname{det} K_{1}^{-1}=\operatorname{det} K_{2}^{-1}=\left(e^{i \sqrt{\lambda} a}-9 e^{-i \sqrt{\lambda} a}\right)^{-4} \\
& \quad \cdot e^{-4 i \sqrt{\lambda} a}\left[\xi\left(\xi \eta^{2}-64\right)(\xi-8)^{2}+16 \eta\left(-256-128 \xi+44 \xi^{2}-3 \xi^{3}\right)\right],
\end{aligned}
$$

where $\xi=\exp \{2 i \sqrt{\lambda} a\}-1$ and $\eta=\exp \{2 i \sqrt{\lambda} b\}-1$. Obviously these determinants vanish if $e^{2 i \sqrt{\lambda} a}=e^{2 i \sqrt{\lambda} b}=1$. One can show that there are no other zeros. Note that the embedded eigenvalues of the operator $-\Delta(a, b)$ are determined by the equation $e^{2 i \sqrt{\lambda} a}=e^{2 i \sqrt{\lambda} b}=1$ such that for incommensurable $a$ and $b$ there are no embedded eigenvalues. 
For $e^{2 i \sqrt{\lambda} a}=e^{2 i \sqrt{\lambda} b}=1$ the matrix $S_{a}(\lambda)$ is not compatible with $V(\underline{b}) S_{a}(\lambda) V(\underline{b})$ and

$$
K_{1}^{-1}=K_{2}^{-1}=\frac{1}{2}\left(\begin{array}{cc}
1 & \pm 1 \\
\pm 1 & 1
\end{array}\right)
$$

where \pm 1 corresponds to $\exp \{i \sqrt{\lambda} a\}= \pm 1$. Obviously $\operatorname{Ker} K_{1}^{-1}=\operatorname{Ker} K_{2}^{-1}$ is the subspace spanned by the vector $(1, \mp 1)^{T}$. Further,

$$
\left(S_{a}(\lambda)\right)_{12}\left(\begin{array}{c}
1 \\
\mp 1
\end{array}\right)=\frac{1}{2}\left(\begin{array}{cc}
1 & \pm 1 \\
\pm 1 & 1
\end{array}\right)\left(\begin{array}{c}
1 \\
\mp 1
\end{array}\right)=0
$$

and

$$
\left(V(\underline{b}) S_{a}(\lambda) V(\underline{b})\right)_{12}\left(\begin{array}{c}
1 \\
\mp 1
\end{array}\right)=\frac{1}{2} e^{i \sqrt{\lambda} b}\left(\begin{array}{cc}
1 & \pm 1 \\
\pm 1 & 1
\end{array}\right)\left(\begin{array}{c}
1 \\
\mp 1
\end{array}\right)=0 .
$$

Thus, as proved in Section \$, the generalized star product is well defined also in the case when the matrix $S_{a}(\lambda)$ is not compatible with $V(\underline{b}) S_{a}(\lambda) V(\underline{b})$.

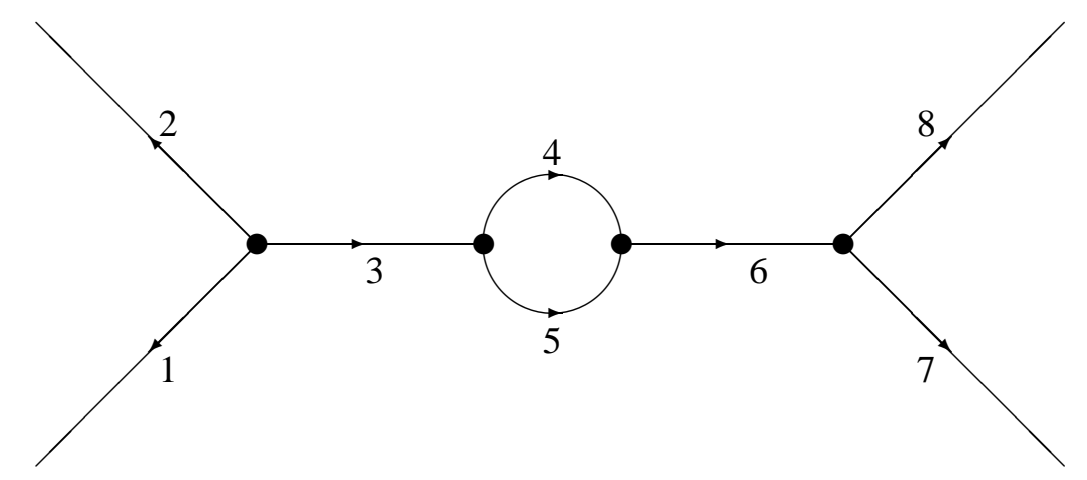

FIG. 5. The graph from Example 4.3. The arrows show the positive direction for every edge. The edges 3 and 6 have the length $a$ and the edges 4 and 5 the length $b$.

Example 4.4. Consider the graph depicted in Fig. 5 where the length of the edges 3 and 6 equals $a$ and the length of the edges 4 and 5 equals $b$. Let the boundary conditions be given by

$$
\begin{array}{r}
\psi_{1}(0)=\psi_{2}(0)=\psi_{3}(0), \\
\psi_{4}(0)=\psi_{5}(0)=\psi_{3}(a), \\
\psi_{1}^{\prime}(0)+\psi_{2}^{\prime}(0)+\psi_{3}^{\prime}(0)=0, \\
\psi_{4}^{\prime}(0)+\psi_{5}^{\prime}(0)-\psi_{3}^{\prime}(a)=0, \\
\psi_{4}(b)=\psi_{5}(b)=\psi_{6}(0), \\
\psi_{6}(a)=\psi_{7}(0)=\psi_{8}(0), \\
-\psi_{4}^{\prime}(b)-\psi_{5}^{\prime}(b)+\psi_{6}^{\prime}(0)=0, \\
-\psi_{6}^{\prime}(a)+\psi_{7}^{\prime}(0)+\psi_{8}^{\prime}(0)=0,
\end{array}
$$

Obviously they define a self-adjoint operator which we denote by $\Delta(a, b)$. The scattering matrix corresponding to this operator (as defined by (2.3) and (2.4)) will be denoted by $S_{a, b}(\lambda)$. To determine this $4 \times 4$ matrix we first consider the graph depicted in Fig. 4 where the length of the edge 3 is supposed to be equal a. The boundary conditions (4.23) determine the self-adjoint operator. The corresponding scattering matrix $S_{a}(\lambda)$ can be obtained from (4.24) by means of 


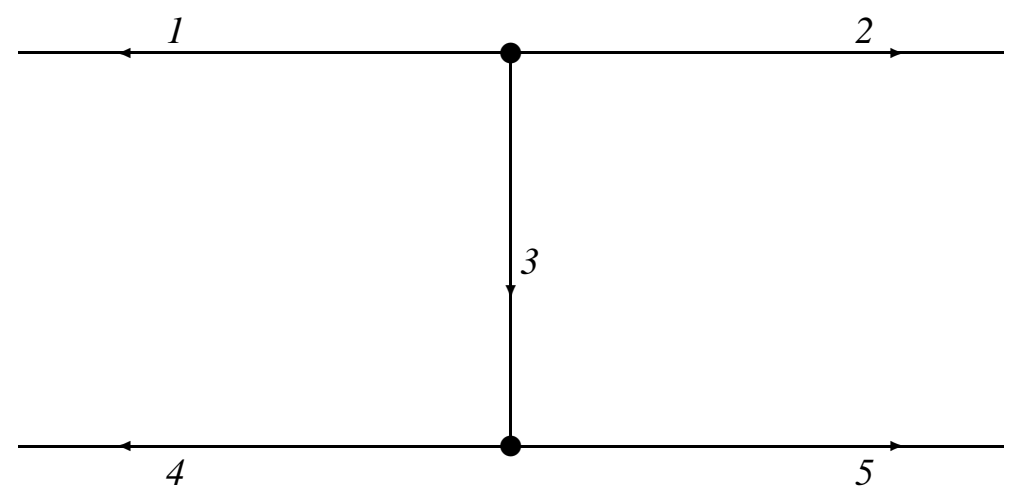

FIG. 6. The same graph as in Fig. $\bigoplus$ but with different ordering of the external lines.

the permutation of its lines and columns thus giving

$$
\begin{gathered}
S_{a}(\lambda)=\left(e^{i \sqrt{\lambda} a}-9 e^{-i \sqrt{\lambda} a}\right)^{-1} \\
.\left(\begin{array}{cccc}
e^{i \sqrt{\lambda} a}+3 e^{-i \sqrt{\lambda} a} & 2\left(e^{i \sqrt{\lambda} a}-3 e^{-i \sqrt{\lambda} a}\right) & -4 & -4 \\
2\left(e^{i \sqrt{\lambda} a}-3 e^{-i \sqrt{\lambda} a}\right) & e^{i \sqrt{\lambda} a}+3 e^{-i \sqrt{\lambda} a} & -4 & -4 \\
-4 & -4 & e^{i \sqrt{\lambda} a}+3 e^{-i \sqrt{\lambda} a} & 2\left(e^{i \sqrt{\lambda} a}-3 e^{-i \sqrt{\lambda} a}\right) \\
-4 & -4 & 2\left(e^{i \sqrt{\lambda} a}-3 e^{-i \sqrt{\lambda} a}\right) & e^{i \sqrt{\lambda} a}+3 e^{-i \sqrt{\lambda} a}
\end{array}\right) .
\end{gathered}
$$

By Theorem 4.1 the scattering matrix $S_{a, b}(\lambda)$ is given by

$$
S_{a, b}(\lambda)=S_{a}(\lambda) *_{2} V(\underline{b}) S_{a}(\lambda) V(\underline{b}),
$$

where

$$
V(\underline{b})=\operatorname{diag}\left(e^{i \sqrt{\lambda} b}, e^{i \sqrt{\lambda} b}, 1,1\right), \quad \underline{b}=(b, b) .
$$

We now compute the $2 \times 2$ matrices $K_{1}$ and $K_{2}$ entering the definition (3.4) of the generalized star product, thus obtaining

$$
\begin{aligned}
& K_{1}^{-1}=K_{2}^{-1}=\left(e^{i \sqrt{\lambda} a}-9 e^{-i \sqrt{\lambda} a}\right)^{-2} L, \\
& (L)_{11}=(L)_{22}=e^{2 i \sqrt{\lambda} a}\left(1-5 e^{2 i \sqrt{\lambda} b}\right)+9 e^{-2 i \sqrt{\lambda} a}\left(9-5 e^{2 i \sqrt{\lambda} b}\right)-18\left(1-e^{2 i \sqrt{\lambda} b}\right), \\
& (L)_{12}=(L)_{21}=-4 e^{2 i \sqrt{\lambda} b}\left(e^{2 i \sqrt{\lambda} a}-9 e^{-2 i \sqrt{\lambda} a}\right) .
\end{aligned}
$$

From this it follows that

$$
\begin{aligned}
& \operatorname{det} K_{1}^{-1}=\operatorname{det} K_{2}^{-1}=\left(e^{i \sqrt{\lambda} a}-9 e^{-i \sqrt{\lambda} a}\right)^{-4}\left[e^{4 i \sqrt{\lambda} a}\left(1-10 e^{2 i \sqrt{\lambda} b}+9 e^{4 i \sqrt{\lambda} b}\right)\right. \\
&+9^{3} e^{-4 i \sqrt{\lambda} a}\left(9-10 e^{2 i \sqrt{\lambda} b}+e^{4 i \sqrt{\lambda} b}\right)-36 e^{2 i \sqrt{\lambda} a}\left(1-6 e^{2 i \sqrt{\lambda} b}+5 e^{4 i \sqrt{\lambda} b}\right) \\
&\left.-18^{2} e^{-2 i \sqrt{\lambda} a}\left(9-14 e^{2 i \sqrt{\lambda} b}+5 e^{4 i \sqrt{\lambda} b}\right)+18\left(27-86 e^{2 i \sqrt{\lambda} b}+59 e^{4 i \sqrt{\lambda} b}\right)\right] .
\end{aligned}
$$

Obviously these determinants vanish if $e^{2 i \sqrt{\lambda} b}=1$. One can show that there are no other zeros. Note that the embedded eigenvalues of the operator $-\Delta(a, b)$ are determined by the equation $e^{2 i \sqrt{\lambda} b}=1$.

For $e^{2 i \sqrt{\lambda} b}=1$ the matrices $S_{a}(\lambda)$ and $V(\underline{b}) S_{a}(\lambda) V(\underline{b})$ are not compatible and

$$
K_{1}^{-1}=K_{2}^{-1}=-4 \frac{e^{2 i \sqrt{\lambda} a}-9 e^{-2 i \sqrt{\lambda} a}}{\left(e^{i \sqrt{\lambda} a}-9 e^{-i \sqrt{\lambda} a}\right)^{2}}\left(\begin{array}{ll}
1 & 1 \\
1 & 1
\end{array}\right) .
$$


Obviously $\operatorname{Ker} K_{1}^{-1}=\operatorname{Ker} K_{2}^{-1}$ is the subspace spanned by the vector $(1,-1)^{T}$. Further,

$$
\left(S_{a}(\lambda)\right)_{12}\left(\begin{array}{c}
1 \\
-1
\end{array}\right)=-4\left(\begin{array}{ll}
1 & 1 \\
1 & 1
\end{array}\right)\left(\begin{array}{c}
1 \\
-1
\end{array}\right)=0
$$

and

$$
\left(V(\underline{b}) S_{a}(\lambda) V(\underline{b})\right)_{12}\left(\begin{array}{c}
1 \\
-1
\end{array}\right)=-4 e^{i \sqrt{\lambda} b}\left(\begin{array}{ll}
1 & 1 \\
1 & 1
\end{array}\right)\left(\begin{array}{c}
1 \\
-1
\end{array}\right)=0 .
$$

Thus, as proved in Section 3 , the generalized star product is well defined also in the case when the matrices $S_{a}(\lambda)$ and $V(\underline{b}) S_{a}(\lambda) V(\underline{b})$ are not compatible.

As already discussed in [42] multiple application of (4.2) to an arbitrary graph allows one by complete induction on the number of vertices to calculate its scattering matrix from the scattering matrices corresponding to single-vertex graphs. If these single vertex graphs contain no tadpoles, i.e. internal lines starting and ending at the same vertex, then (4.2) give a complete explicit construction of the scattering matrix in terms of the scattering matrices for single vertex graphs. In case when a resulting single-vertex graph contains tadpoles we proceed as follows. Let the graph $\Gamma$ have one vertex, $n$ external lines and $m$ tadpoles of lengths $a_{i}$. To calculate the scattering matrix of $\Gamma$ we insert an extra vertex on each of the internal lines (for definiteness, say, at $x=a_{i} / 2$ ). At these new vertices we impose trivial boundary conditions corresponding to continuous differentiability at this point. With these new vertices we may now repeat our previous procedure. Thus in the end we arrive at graphs with one vertex only and no tadpoles.

\section{Special CASE $n_{1}=n_{2}=2 p$ : TRANSFer Matrices}

This section is devoted to the construction of the transfer matrix for Schrödinger operators on graphs with an even number of external lines. The transfer matrix formalism for general Schrödinger operators on the line is well known (see e.g. [19]). Its relation to the scattering matrix is discussed in e.g. [71, 41].

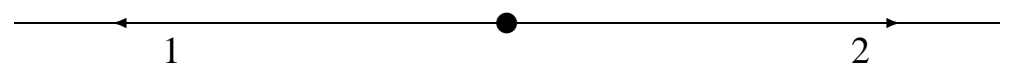

FIG. 7. The graph with $n=2$ and $m=0$.

We start with the simplest example of a Laplace operator on the graph with $n=2$ and $m=0$ (see Fig. 7) which is equivalent to a Schrödinger operator on the line with point interaction. The boundary conditions given by the relation

$$
\left(\begin{array}{l}
\psi_{2}(0) \\
\psi_{2}^{\prime}(0)
\end{array}\right)=e^{i \mu}\left(\begin{array}{ll}
a & b \\
c & d
\end{array}\right)\left(\begin{array}{c}
\psi_{1}(0) \\
-\psi_{1}^{\prime}(0)
\end{array}\right)
$$

where the matrix

$$
\left(\begin{array}{ll}
a & b \\
c & d
\end{array}\right) \in \mathrm{SL}(2, \mathbb{R})
$$

and $\mu$ is real, lead to self-adjoint Laplacians (see [142, 68, 16, 49, 3]). Conversely, from the viewpoint of the von Neumann extension theory (see e.g. [59]) relation (5.1) describes almost all (with respect to the Haar measure on $U(2)$ ) self-adjoint Laplacians $\Delta(A, B)$. If $\exp \{2 i \mu\}=1$ the operator $\Delta(A, B)$ is real, i.e. commutes with complex conjugation. In particular, the choice $a-1=d-1=b=0, \exp \{2 i \mu\}=1$ corresponds to the $\delta$-potential of strength $c$ (see e.g. [纤]). 
By definition the transfer matrix is a $2 \times 2$ matrix $M(\lambda) \in \mathrm{U}(1) \times \mathrm{SL}(2, \mathbb{R})$ satisfying

$$
M(\lambda)\left(\begin{array}{c}
\psi_{1}(0) \\
\psi_{1}^{\prime}(0)
\end{array}\right)=\left(\begin{array}{c}
\psi_{2}(0) \\
-\psi_{2}^{\prime}(0)
\end{array}\right)
$$

In fact, in the case at hand it is given explicitly as follows

$$
M(\lambda)=e^{i \mu}\left(\begin{array}{ll}
a & b \\
c & d
\end{array}\right)
$$

If $\exp \{2 i \mu\}=1$ the matrix $M(\lambda)$ is unimodular, i.e. $M(\lambda) \in \mathrm{SL}(2 ; \mathbb{R})$.

The transfer matrix possesses the following equivalent description. Any solution of the Schrödinger equation with the operator $-\Delta(A, B)$ for the energy $\lambda>0$ has the form

$$
\begin{aligned}
& u_{1}(x)=a_{1} e^{i \sqrt{\lambda} x}+b_{1} e^{-i \sqrt{\lambda} x}, \\
& u_{2}(x)=a_{2} e^{i \sqrt{\lambda} x}+b_{2} e^{-i \sqrt{\lambda} x} .
\end{aligned}
$$

From this and (5.2) it follows that there is a matrix $\Lambda(\lambda) \in U(1) \times S U(1,1) \subset U(1) \times S L(2 ; \mathbb{C})$ (with the inclusion in the group-theoretical sense) such that

$$
\Lambda(\lambda)\left(\begin{array}{l}
a_{1} \\
b_{1}
\end{array}\right)=\left(\begin{array}{l}
b_{2} \\
a_{2}
\end{array}\right)
$$

and

$$
M(\lambda)=\left(\begin{array}{cc}
1 & 1 \\
i \sqrt{\lambda} & -i \sqrt{\lambda}
\end{array}\right) \Lambda(\lambda)\left(\begin{array}{cc}
1 & 1 \\
i \sqrt{\lambda} & -i \sqrt{\lambda}
\end{array}\right)^{-1} .
$$

For $\lambda>0$ the matrix $\Lambda(\lambda)$ is related to the scattering matrix

$$
S_{A, B}(\lambda)=\left(\begin{array}{ll}
R(\lambda) & T_{1}(\lambda) \\
T_{2}(\lambda) & L(\lambda)
\end{array}\right)
$$

by the relation

$$
\Lambda(\lambda)=\left(\begin{array}{cc}
\frac{1}{T_{2}(\lambda)} & -\frac{R(\lambda)}{T_{2}(\lambda)} \\
\frac{L(\lambda)}{T_{2}(\lambda)} & \frac{T_{1}(\lambda)}{\left|T_{2}(\lambda)\right|^{2}}
\end{array}\right),
$$

where

$$
\begin{aligned}
T_{1}(\lambda) & =2 e^{i \mu}(a-i b \sqrt{\lambda}+i c / \sqrt{\lambda}+d)^{-1}, \\
T_{2}(\lambda) & =2 e^{-i \mu}(a-i b \sqrt{\lambda}+i c / \sqrt{\lambda}+d)^{-1}, \\
R(\lambda) & =(a-i b \sqrt{\lambda}+i c / \sqrt{\lambda}+d)^{-1}(a-i b \sqrt{\lambda}-i c / \sqrt{\lambda}-d), \\
L(\lambda) & =(a-i b \sqrt{\lambda}+i c / \sqrt{\lambda}+d)^{-1}(-a-i b \sqrt{\lambda}-i c / \sqrt{\lambda}+d) .
\end{aligned}
$$

Note that $T_{1}(\lambda)=T_{2}(\lambda)$ for all $\lambda>0$ if the operator $\Delta(A, B)$ is real, i.e. $\exp \{2 i \mu\}=1$. This is in analogy with Schrödinger operators on the line with potentials which are necessarily real (see e.g. [29, 21]).

The factorization rule from Example 4.2 can now be written in the form

$$
\Lambda(\lambda)=\Lambda^{(1)}(\lambda) U(a) \Lambda^{(2)}(\lambda) U(a)^{-1},
$$

where

$$
U(a)=\left(\begin{array}{cc}
e^{-i \sqrt{\lambda} a} & 0 \\
0 & e^{i \sqrt{\lambda} a}
\end{array}\right) .
$$

The relation (5.4) is the special case of the well-known factorization formula [1, 57, 58, 47, 62, 64. 65] applied to the Laplacian on a line with point interaction. 
It is easy to realize that the transfer matrix cannot be defined for arbitrary boundary conditions. For instance, the Dirichlet $\left(\psi_{2}(0+)=\psi_{1}(0+)=0\right)$ or Neuman $\left(\psi_{2}^{\prime}(0+)=\psi_{1}^{\prime}(0+)=0\right)$ or mixed $\left(\psi_{2}(0+)+k_{2} \psi_{2}^{\prime}(0+)=\psi_{1}(0+)+k_{1} \psi_{1}^{\prime}(0+)=0\right)$ boundary conditions introduce the decoupling $\Delta(A, B)=\Delta_{1} \oplus \Delta_{2}$, where $\Delta_{j}, j=1,2$ are the Laplacians on $L^{2}(0, \infty)$ with corresponding boundary conditions. Recall, however, that the scattering matrix is well defined even in these cases. The composition rule (4.22) (see Example 4.2) remains valid.

Now we consider an arbitrary graph $\Gamma$ with an even number of external lines $n=2 p$. We enumerate the external lines in an arbitrary but fixed order. The external part of an arbitrary solution of the Schrödinger equation with $-\Delta(A, B)$ at the energy $\lambda>0$ has the form

$$
u_{j}(x)=a_{j} e^{i \sqrt{\lambda} x}+b_{j} e^{-i \sqrt{\lambda} x}, \quad j=1, \ldots, n .
$$

We define the transfer matrix

$$
\Lambda(\lambda)\left(\begin{array}{c}
a_{1} \\
\vdots \\
a_{p} \\
b_{1} \\
\vdots \\
b_{p}
\end{array}\right)=\left(\begin{array}{c}
b_{p+1} \\
\vdots \\
b_{n} \\
a_{p+1} \\
\vdots \\
a_{n}
\end{array}\right) .
$$

To prove that $\Lambda(\lambda)$ is correctly defined it suffices to show that for arbitrary constants $\left(a_{j}, b_{j}\right)$, $j=1, \ldots, p$ there is a solution to the Schrödinger equation with the operator $-\Delta(A, B)$ whose external part has the form (5.5) and this solution is unique up to its internal part. The external part of any solution to the Schrödinger equation with the operator $-\Delta(A, B)$ is a linear combination of the columns of the matrix-valued function

$$
\Psi(x, \lambda)=e^{-i \sqrt{\lambda} x} \mathbb{I}+e^{i \sqrt{\lambda} x} S(\lambda) .
$$

Thus, the columns of (5.7) have to satisfy $(5.6)$, i.e.

$$
\Lambda(\lambda)\left(\begin{array}{cc}
S_{11}(\lambda) & S_{12}(\lambda) \\
\mathbb{I} & 0
\end{array}\right)=\left(\begin{array}{cc}
0 & \mathbb{I} \\
S_{21}(\lambda) & S_{22}(\lambda)
\end{array}\right),
$$

where the $p \times p$ block notation is adopted. Writing $\Lambda(\lambda)$ as

we obtain

$$
\Lambda(\lambda)=\left(\begin{array}{ll}
\Lambda_{11}(\lambda) & \Lambda_{12}(\lambda) \\
\Lambda_{21}(\lambda) & \Lambda_{22}(\lambda)
\end{array}\right)
$$

$$
\begin{aligned}
& \Lambda_{11}(\lambda) S_{11}(\lambda)+\Lambda_{12}(\lambda)=0, \\
& \Lambda_{11}(\lambda) S_{12}(\lambda)=\mathbb{I}, \\
& \Lambda_{21}(\lambda) S_{11}(\lambda)+\Lambda_{22}(\lambda)=S_{21}(\lambda), \\
& \Lambda_{21}(\lambda) S_{12}(\lambda)=S_{22}(\lambda) .
\end{aligned}
$$

Let us suppose that det $S_{12}(\lambda) \neq 0$. Then

$$
\begin{aligned}
\Lambda_{11}(\lambda)=S_{12}(\lambda)^{-1}, & & \Lambda_{12}(\lambda)=-S_{12}(\lambda)^{-1} S_{11}(\lambda), \\
\Lambda_{21}(\lambda)=S_{22}(\lambda) S_{12}(\lambda)^{-1}, & & \Lambda_{22}(\lambda)=S_{21}(\lambda)-S_{22}(\lambda) S_{12}(\lambda)^{-1} S_{11}(\lambda) .
\end{aligned}
$$

Thus, we proved that for det $S_{12}(\lambda) \neq 0$ the transfer matrix exists and has the form

$$
\Lambda(\lambda)=\left(\begin{array}{cc}
S_{12}(\lambda)^{-1} & -S_{12}(\lambda)^{-1} S_{11}(\lambda) \\
S_{22}(\lambda) S_{12}(\lambda)^{-1} & S_{21}(\lambda)-S_{22}(\lambda) S_{12}(\lambda)^{-1} S_{11}(\lambda)
\end{array}\right) .
$$

Also, its definition (5.6) immediately leads to the following factorization formula

$$
\Lambda(\lambda)=\Lambda^{(1)}(\lambda) U(\underline{a}) \Lambda^{(2)}(\lambda) U(\underline{a})^{-1},
$$


where the diagonal unitary matrix $U(\underline{a})$ is given by

$$
U(\underline{a})=\left(\begin{array}{cc}
e^{-i \sqrt{\lambda} \underline{a}} & 0 \\
0 & e^{i \sqrt{\lambda} \underline{a}}
\end{array}\right) .
$$

Note that formal arguments based on the superposition principle leading to (5.10) have appeared earlier in [20]. As for related results we mention that in [50] it was shown that the transfer matrix of a Schrödinger operator on the line with a matrix-valued potential can be written in the form (5.9).

Lemma 5.1. If $\operatorname{det} S_{12}(\lambda) \neq 0$ then $\Lambda(\lambda) \in \mathrm{U}(p, p)$.

Proof. Obviously the coefficients $a_{1}, \ldots, a_{n}, b_{1}, \ldots, b_{n}$ in (5.5) satisfy the relation

$$
\left(\begin{array}{c}
a_{1} \\
\vdots \\
a_{n}
\end{array}\right)=S(\lambda)\left(\begin{array}{c}
b_{1} \\
\vdots \\
b_{n}
\end{array}\right) .
$$

From the unitarity of the scattering matrix it follows that

$$
\left|a_{1}\right|^{2}+\ldots+\left|a_{n}\right|^{2}=\left|b_{1}\right|^{2}+\ldots+\left|b_{n}\right|^{2},
$$

or, equivalently,

$$
\left|a_{1}\right|^{2}+\ldots+\left|a_{p}\right|^{2}-\left|b_{1}\right|^{2}-\left|b_{p}\right|^{2}=\left|b_{p+1}\right|^{2}+\ldots+\left|b_{n}\right|^{2}-\left|a_{p+1}\right|^{2}-\ldots-\left|a_{n}\right|^{2} .
$$

This relation and (5.6) complete the proof of the lemma.

Let us summarize the above results of the present Section:

Theorem 5.2. If $\operatorname{det} S_{12}(\lambda) \neq 0$ then the transfer matrix $\Lambda(\lambda) \in \mathrm{U}(p, p)$ as given by (5.9) exists such that for an arbitrary $\left(a_{1}, \ldots, a_{p}, b_{1}, \ldots, b_{p}\right) \in \mathbb{C}^{n}$ there is a solution of the Schrödinger equation with $-\Delta(A, B)$ at the energy $\lambda>0$ whose external part has the form (5.5) and the coefficients $\left(b_{p+1}, \ldots, b_{n}, a_{p+1}, \ldots, a_{n}\right) \in \mathbb{C}^{n}$ are given by (5.6). The composition rule for the scattering matrices (4.2) is equivalent to the multiplication formula (5.10) for the transfer matrices.

In addition for real operators we have

Theorem 5.3. If the operator $\Delta(A, B, \underline{a})$ is real and if in addition $\operatorname{det} S_{12}(\lambda) \neq 0$ then $\Lambda(\lambda) \in$ $\mathrm{SU}(p, p) \subset \mathrm{SL}(2 p ; \mathbb{C})$.

Proof. From the well-known determinant formula for block matrices (see e.g. [30, Section II.5])

$$
\operatorname{det}\left(\begin{array}{ll}
A_{11} & A_{12} \\
A_{21} & A_{22}
\end{array}\right)=\operatorname{det} A_{11} \operatorname{det}\left(A_{22}-A_{21} A_{11}^{-1} A_{12}\right)
$$

which follows from the decomposition

$$
\left(\begin{array}{ll}
A_{11} & A_{12} \\
A_{21} & A_{22}
\end{array}\right)=\left(\begin{array}{ll}
A_{11} & 0 \\
A_{21} & \mathbb{I}
\end{array}\right)\left(\begin{array}{cc}
\mathbb{I} & A_{11}^{-1} A_{12} \\
0 & A_{22}-A_{21} A_{11}^{-1} A_{12}
\end{array}\right),
$$

it follows that

$$
\operatorname{det} \Lambda(\lambda)=\frac{\operatorname{det} S_{21}(\lambda)}{\operatorname{det} S_{12}(\lambda)}
$$

By Theorem 2.2 we have $S_{12}(\lambda)^{T}=S_{21}(\lambda)$ and thus $\operatorname{det} \Lambda(\lambda)=1$.

We turn now to a discussion of the assumption det $S_{12}(\lambda) \neq 0$. For the scattering matrix of the graph depicted in Fig. 4 with the boundary conditions (4.23) (see Example 4.3) $\operatorname{det}\left(S_{a}(\lambda)\right)_{12}=0$ for all $\lambda>0$. 
Theorem 5.4. Suppose that $\operatorname{det} S_{12}(\lambda)=0$. Then the transfer matrix $\Lambda(\lambda)$ exists such that for arbitrary

$$
\left(a_{1}, \ldots, a_{p}, b_{1}, \ldots, b_{p}\right) \in \operatorname{Ran}\left(\begin{array}{cc}
\mathbb{I} & 0 \\
0 & P_{\left(\operatorname{Ker} S_{12}(\lambda)\right)^{\perp}}
\end{array}\right) \subset \mathbb{C}^{n}
$$

there is a solution of the Schrödinger equation with $-\Delta(A, B, \underline{a})$ at energy $\lambda>0$ whose external part has the form (5.5) and the coefficients $\left(b_{p+1}, \ldots, b_{n}, a_{p+1}, \ldots, a_{n}\right) \in \mathbb{C}^{n}$ are given by (5.6).

Proof. The external part of any solution to the Schrödinger equation with the operator $-\Delta(A, B, \underline{a})$ satisfying the conditions of the theorem is a linear combination of the columns of the matrixvalued function

$$
\Psi(x, \lambda)\left(\begin{array}{cc}
\mathbb{I} & 0 \\
0 & P_{\left(\operatorname{Ker} S_{12}(\lambda)\right)^{\perp}}
\end{array}\right),
$$

where $\Psi(x, \lambda)$ is given by (5.7). Thus, the columns of (5.12) have to satisfy (5.6), i.e.

$$
\left(\begin{array}{ll}
\Lambda_{11}(\lambda) & \Lambda_{12}(\lambda) \\
\Lambda_{21}(\lambda) & \Lambda_{22}(\lambda)
\end{array}\right)\left(\begin{array}{cc}
S_{11}(\lambda) & S_{12}(\lambda) P_{\left(\operatorname{Ker} S_{12}(\lambda)\right)^{\perp}} \\
\mathbb{I} & 0
\end{array}\right)=\left(\begin{array}{cc}
0 & P_{\left(\operatorname{Ker} S_{12}(\lambda)\right)^{\perp}} \\
S_{21}(\lambda) & S_{22}(\lambda) P_{\left(\operatorname{Ker} S_{12}(\lambda)\right)^{\perp}}
\end{array}\right) .
$$

The solution of this equation can be wiritten in the form

$$
\begin{array}{cl}
\Lambda_{11}(\lambda)=S_{12}(\lambda)^{\star}, & \Lambda_{12}(\lambda)=-S_{12}(\lambda)^{\star} S_{11}(\lambda), \\
\Lambda_{21}(\lambda)=S_{22}(\lambda) S_{12}(\lambda)^{\star}, & \Lambda_{22}=S_{21}(\lambda)-S_{22}(\lambda) S_{12}(\lambda)^{\star} S_{11}(\lambda),
\end{array}
$$

where $\star$ stands for the Penrose-Moore pseudoinverse.

Note that any vector of the form $(c, 0)^{T}$ with $c \in \operatorname{Ker} S_{12}(\lambda)$ satisfies $\Lambda(\lambda)\left(\begin{array}{l}c \\ 0\end{array}\right)=0$. Thus $\operatorname{det} \Lambda(\lambda)=0$.

Inspection of the proof of Theorem 5.4 shows that the transfer matrix cannot be extended to a subspace larger than

$$
\operatorname{Ran}\left(\begin{array}{cc}
\mathbb{I} & 0 \\
0 & P_{\left(\operatorname{Ker} S_{12}(\lambda)\right)^{\perp}}
\end{array}\right)
$$

If $\operatorname{det} \Lambda^{(1)}(\lambda)=\operatorname{det} \Lambda^{(2)}(\lambda)=0$ then $\operatorname{Ran} U(\underline{a}) \Lambda^{(2)}(\lambda) U(\underline{a})$ and $\operatorname{Ker} \Lambda^{(1)}(\lambda)$ may have a nontrivial overlap and therefore the multiplication formula (5.10) does not hold in this case.

Example 5.5. Consider the graph depicted in Fig. $⿴$ with the boundary conditions from Example 4.3. For all $\lambda \in \mathbb{R}_{+}$such that $e^{2 i \sqrt{\lambda} a}=1$ we have that $\operatorname{Ker} S_{12}(\lambda)$ is nontrivial and

$$
\begin{gathered}
P_{\operatorname{Ker} S_{12}(\lambda)}=\frac{1}{2}\left(\begin{array}{cc}
1 & \mp 1 \\
\mp 1 & 1
\end{array}\right), \\
P_{\left(\operatorname{Ker} S_{12}(\lambda)\right)^{\perp}}=\frac{1}{2}\left(\begin{array}{cc}
1 & \pm 1 \\
\pm 1 & 1
\end{array}\right),
\end{gathered}
$$

where \pm 1 corresponds to $\exp \{i \sqrt{\lambda} a\}= \pm 1$. Suppose that

or, equivalently,

$$
\left(a_{1}, a_{2}, b_{1}, b_{2}\right)^{T} \notin \operatorname{Ran}\left(\begin{array}{cc}
\mathbb{I} & 0 \\
0 & P_{\left(\operatorname{Ker} S_{12}(\lambda)\right)^{\perp}}
\end{array}\right),
$$

$$
\left(a_{1}, a_{2}, b_{1}, b_{2}\right)^{T} \in \operatorname{Ran}\left(\begin{array}{cc}
0 & 0 \\
0 & P_{\operatorname{Ker} S_{12}(\lambda)}
\end{array}\right) .
$$

In particular, we can choose

$$
a_{1}=a_{2}=0, \quad b_{1}=1, \quad b_{2}=\mp 1 .
$$

It is easy to check that there is no solution to the Schrödinger equation with these boundary conditions. 
Example 5.6. Consider the graph depicted in Fig. 6 with the boundary conditions as in Example 4.4. $\operatorname{Ker} S_{12}(\lambda)$ is nontrivial for all $\lambda \in \mathbb{R}_{+}$and

$$
P_{\text {Ker } S_{12}(\lambda)}=\frac{1}{2}\left(\begin{array}{cc}
1 & -1 \\
-1 & 1
\end{array}\right), \quad P_{\left(\operatorname{Ker} S_{12}(\lambda)\right)^{\perp}}=\frac{1}{2}\left(\begin{array}{ll}
1 & 1 \\
1 & 1
\end{array}\right) .
$$

Suppose again that

$$
\left(a_{1}, a_{2}, b_{1}, b_{2}\right)^{T} \notin \operatorname{Ran}\left(\begin{array}{cc}
\mathbb{I} & 0 \\
0 & P_{\left(\operatorname{Ker} S_{12}(\lambda)\right)^{\perp}}
\end{array}\right)
$$

and choose

$$
a_{1}=a_{2}=0, \quad b_{1}=1, \quad b_{2}=1 .
$$

Again it is easy to check that there is no solution to the Schrödinger equation with these boundary conditions.

The statement converse to Theorem 5.2 immediately follows from Theorem 5.4.

Theorem 5.7. If the transfer matrix $\Lambda(\lambda)$ exists in the sense of Theorem 5.2 then $\operatorname{det} S_{12}(\lambda) \neq 0$ and the corresponding scattering matrix is given by

$$
S(\lambda)=\left(\begin{array}{cc}
-\Lambda_{11}(\lambda)^{-1} \Lambda_{12}(\lambda) & \Lambda_{11}(\lambda)^{-1} \\
\Lambda_{22}(\lambda)-\Lambda_{21}(\lambda) \Lambda_{11}(\lambda)^{-1} \Lambda_{12}(\lambda) & \Lambda_{21}(\lambda) \Lambda_{11}(\lambda)^{-1}
\end{array}\right) .
$$

Proof. Suppose that det $S_{12}(\lambda)=0$. Then by Theorem 5.4 we get $\operatorname{det} \Lambda(\lambda)=0$, which is a contradiction. Thus, det $S_{12}(\lambda) \neq 0$ and therefore by Theorem 5.2 det $\Lambda(\lambda) \neq 0$. The representation (5.13) follows from (5.8).

\section{APPENDIX A}

Here we give the proof of Theorem 3.6 which claims that for arbitrary unitary matrices $U^{(1)}$, $U^{(2)}$, and $V$ the matrix $U=U^{(1)} *_{V} U^{(2)}$ defined by (3.4) is unitary. As already noted in [42] it suffices to prove only the relations

$$
\begin{aligned}
& U_{11}{ }^{*} U_{11}+U_{21}{ }^{*} U_{21}=\mathbb{I}, \\
& U_{11}{ }^{*} U_{12}+U_{21}{ }^{*} U_{22}=0 .
\end{aligned}
$$

The remaining relations

$$
\begin{aligned}
& U_{12}{ }^{*} U_{12}+U_{22}{ }^{*} U_{22}=\mathbb{I}, \\
& U_{12}{ }^{*} U_{11}+U_{22}{ }^{*} U_{21}=0 .
\end{aligned}
$$

follow immediately from A.1. To see this for an arbitrary unitary matrix $U$ we define an involutive map $U \mapsto U^{\tau}$ given as

$$
U=\left(\begin{array}{ll}
U_{11} & U_{12} \\
U_{21} & U_{22}
\end{array}\right) \quad \mapsto \quad U^{\tau}=\left(\begin{array}{ll}
U_{22} & U_{21} \\
U_{12} & U_{11}
\end{array}\right)
$$

Direct calculations show that the following "transposition law"

$$
U^{\tau}=U^{(2)^{\tau}} *_{V^{*}} U^{(1)^{\tau}}
$$

holds whenever $U=U^{(1)} *_{V} U^{(2)}$. Assume that (A.1) holds for arbitrary unitary $U$. Replacing the matrix $U$ by $U^{\tau}$ given by (A.3) transforms the relations (A.1) into (A.2).

By the definition of the generalized star product (3.4) and by the unitarity of $U^{(1)}$ the first of the relations (A.1) is equivalent to

$$
\begin{aligned}
& -U_{21}^{(1)^{*}} U_{21}^{(1)}+U_{21}^{(1)^{*}} V^{*} U_{11}^{(2)^{*}} K_{2}^{*} U_{12}^{(1)^{*}} U_{11}^{(1)}+U_{11}^{(1)^{*}} U_{12}^{(1)} K_{2} U_{11}^{(2)} V U_{21}^{(1)} \\
& \quad+U_{21}^{(1)^{*}} V^{*} U_{11}^{(2)^{*}} K_{2}^{*} U_{12}^{(1)^{*}} U_{12}^{(1)} K_{2} U_{11}^{(2)} V U_{21}^{(1)}+U_{21}^{(1)^{*}} K_{1}^{*} U_{21}^{(2)^{*}} U_{21}^{(2)} K_{1} U_{21}^{(1)}=0 .
\end{aligned}
$$


Since the opposite case was already considered in [42] we further assume that the matrix $U^{(1)}$ is not $V$-compatible with $U^{(2)}$. From Theorem 3.6 it follows that all off-diagonal blocks $U_{12}^{(1)}, U_{21}^{(1)}$, $U_{12}^{(2)}$, and $U_{21}^{(2)}$ are not of maximal rank and thus $\operatorname{Ker} U_{21}^{(1)}$ is nontrivial. Let $d_{i}, 1 \leq i \leq k=\operatorname{dim} \operatorname{Ker} U_{21}^{(1)}$ be an arbitrary basis in $\operatorname{Ker} U_{21}^{(1)}$. From the unitarity of the matrix $U^{(1)}$ we get $U_{12}^{(1)^{*}} U_{11}^{(1)} d_{i}=0$ for all $1 \leq i \leq k$. Thus

$$
\begin{aligned}
& {\left[-U_{21}^{(1)^{*}} U_{21}^{(1)}+U_{21}^{(1)^{*}} V^{*} U_{11}^{(2)^{*}} K_{2}^{*} U_{12}^{(1)^{*}} U_{11}^{(1)}+U_{11}^{(1)^{*}} U_{12}^{(1)} K_{2} U_{11}^{(2)} V U_{21}^{(1)}\right.} \\
& \left.+U_{21}^{(1)^{*}} V^{*} U_{11}^{(2)^{*}} K_{2}^{*} U_{12}^{(1)^{*}} U_{12}^{(1)} K_{2} U_{11}^{(2)} V U_{21}^{(1)}+U_{21}^{(1)^{*}} K_{1}^{*} U_{21}^{(2)^{*}} U_{21}^{(2)} K_{1} U_{21}^{(1)}\right] d_{i}=0
\end{aligned}
$$

for all $1 \leq i \leq k$. Hence to prove (A.4) it remains to show that

$$
\begin{aligned}
& {\left[-U_{21}^{(1)^{*}} U_{21}^{(1)}+U_{21}^{(1)^{*}} V^{*} U_{11}^{(2)^{*}} K_{2}^{*} U_{12}^{(1)^{*}} U_{11}^{(1)}+U_{11}^{(1)^{*}} U_{12}^{(1)} K_{2} U_{11}^{(2)} V U_{21}^{(1)}\right.} \\
& \left.+U_{21}^{(1)^{*}} V^{*} U_{11}^{(2)^{*}} K_{2}^{*} U_{12}^{(1)^{*}} U_{12}^{(1)} K_{2} U_{11}^{(2)} V U_{21}^{(1)}+U_{21}^{(1)^{*}} K_{1}^{*} U_{21}^{(2)^{*}} U_{21}^{(2)} K_{1} U_{21}^{(1)}\right] d=0
\end{aligned}
$$

for any $d \in\left(\operatorname{Ker} U_{21}^{(1)}\right)^{\perp}=\operatorname{Ran} U_{21}^{(1)^{*}}$. Therefore we set $d=U_{21}^{(1)^{*}} \widetilde{d}$, where $\widetilde{d} \in \mathbb{C}^{p}$ is an arbitrary vector. Thus, the relation (A.5) holds whenever

$$
\begin{aligned}
& {\left[-U_{21}^{(1)^{*}} U_{21}^{(1)} U_{21}^{(1)^{*}}+U_{21}^{(1)^{*}} V^{*} U_{11}^{(2)^{*}} K_{2}^{*} U_{12}^{(1)^{*}} U_{11}^{(1)} U_{21}^{(1)^{*}}+U_{11}^{(1)^{*}} U_{12}^{(1)} K_{2} U_{11}^{(2)} V U_{21}^{(1)} U_{21}^{(1)^{*}}\right.} \\
& \left.+U_{21}^{(1)^{*}} V^{*} U_{11}^{(2)^{*}} K_{2}^{*} U_{12}^{(1)^{*}} U_{12}^{(1)} K_{2} U_{11}^{(2)} V U_{21}^{(1)} U_{21}^{(1)^{*}}+U_{21}^{(1)^{*}} K_{1}^{*} U_{21}^{(2)^{*}} U_{21}^{(2)} K_{1} U_{21}^{(1)} U_{21}^{(1)^{*}}\right] \widetilde{d}=0
\end{aligned}
$$

for all $\tilde{d} \in \mathbb{C}^{p}$.

First we note that by Lemma 3.4 (iv) the relation (A.6) holds for all $\widetilde{d} \in \widetilde{\mathcal{C}}$. Therefore it suffices to prove that $(\mathrm{A} .6)$ holds for all $\widetilde{d} \in \widetilde{\mathcal{C}}^{\perp}$. Observe that in this case by Lemma 3.4 (i) and by the unitarity of the matrices $U^{(1)}$ and $U^{(2)}$ we have

$$
\begin{aligned}
U_{12}^{(1)^{*}} U_{11}^{(1)} U_{21}^{(1)^{*}} \tilde{d} & =-U_{12}^{(1)^{*}} U_{12}^{(1)} U_{22}^{(1)^{*}} \widetilde{d}=-U_{22}^{(1)^{*}} \tilde{d}+U_{22}^{(1)^{*}} U_{22}^{(1)} U_{22}^{(1)^{*}} \widetilde{d} \in \mathcal{B}^{\perp}, \\
U_{11}^{(2)} V U_{21}^{(1)} U_{21}^{(1)^{*}} \widetilde{d} & =U_{11}^{(2)} V \widetilde{d}-U_{11}^{(2)} V U_{22}^{(1)} U_{22}^{(1)} \widetilde{d} \in \widetilde{\mathcal{B}}^{\perp}, \\
U_{21}^{(1)} U_{21}^{(1)^{*}} \tilde{d} & =\widetilde{d}-U_{22}^{(1)} U_{22}^{(1)^{*}} \widetilde{d} \in \widetilde{\mathcal{C}}^{\perp} .
\end{aligned}
$$

To prove the first relation in $\left(\right.$ A.7) it suffices to show that for any $\widetilde{d} \in \widetilde{\mathcal{C}}^{\perp}$ and any $b \in \mathcal{B}$

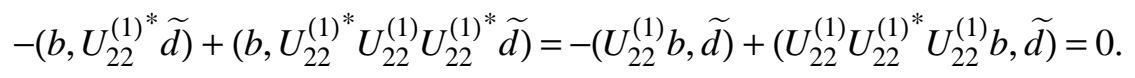

By the definition of $\mathcal{B}$ and by Lemma $3.3 U_{22}^{(1)^{*}} U_{22}^{(1)} b=b$ for any $b \in \mathcal{B}$ which proves A.8). To prove the second relation in (A.7) it suffices to show for any $\widetilde{d} \in \widetilde{\mathcal{C}}^{\perp}$ and any $\widetilde{b} \in \widetilde{\mathcal{B}}$

$$
\begin{aligned}
& \left(\widetilde{b}, U_{11}^{(2)} V \widetilde{d}\right)-\left(\widetilde{b}, U_{11}^{(2)} V U_{22}^{(1)} U_{22}^{(1)^{*}} \widetilde{d}\right)= \\
& \left(V^{*} U_{11}^{(2)} \widetilde{b}, \widetilde{d}\right)-\left(U_{22}^{(1)} U_{22}^{(1)^{*}} V^{*} U_{11}^{(2)^{*}} \widetilde{b}, \widetilde{d}\right)=0 .
\end{aligned}
$$

By Lemma 3.4 (i) $V^{*} U_{11}^{(2)} \widetilde{b} \in \widetilde{\mathcal{C}}$. By the definition of $\widetilde{\mathcal{C}}$ and by Lemma $3.3 U_{22}^{(1)} U_{22}^{(1)^{*}} \widetilde{c}=\widetilde{c}$ for any $\widetilde{c} \in \widetilde{\mathcal{C}}$ which proves (A.9). This also proves that

$$
(\widetilde{c}, \widetilde{d})-\left(\widetilde{c}, U_{22}^{(1)} U_{22}^{(1) * \widetilde{d}}=(\widetilde{c}, \widetilde{d})-\left(U_{22}^{(1)} U_{22}^{(1)^{*}} \widetilde{c}, \widetilde{d}\right)=0\right.
$$

for all $\widetilde{d} \in \widetilde{\mathcal{C}}^{\perp}$ and all $\widetilde{c} \in \widetilde{\mathcal{C}}$ from which the third relation in (A.7) follows. 
From Lemma 3.5 and the definition (3.2) of the matrices $K_{1}$ and $K_{2}$ it follows that

(i) $K_{1}$ maps $\widetilde{\mathfrak{C}}^{\perp}$ onto $\mathrm{C}^{\perp}$ bijectively,

(ii) $K_{1}^{*}$ maps $\mathcal{C}^{\perp}$ onto $\widetilde{\mathcal{C}}^{\perp}$ bijectively,

(iii) $K_{2}$ maps $\widetilde{\mathcal{B}}^{\perp}$ onto $\mathcal{B}^{\perp}$ bijectively,

(iv) $K_{2}^{*}$ maps $\mathcal{B}^{\perp}$ onto $\widetilde{\mathcal{B}}^{\perp}$ bijectively.

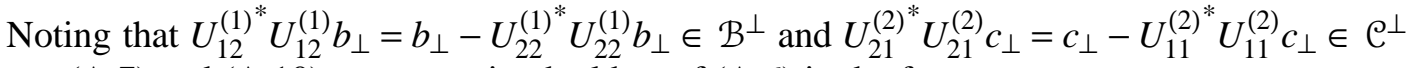
due to (A.7) and A.10) we can write the l.h.s. of (A.6) in the form

$$
\begin{aligned}
& U_{21}^{(1)^{*}}\left[-\mathbb{I}-V^{*} U_{11}^{(2)^{*}} K_{2} U_{22}^{(1)^{*}}-U_{22}^{(1)} K_{2} U_{11}^{(2)} V+V^{*} U_{11}^{(2)^{*}} K_{2}^{*} K_{2} U_{11}^{(2)} V\right. \\
& \left.-V^{*} U_{11}^{(2)^{*}} K_{2}^{*} U_{22}^{(1)^{*}} U_{22}^{(1)} K_{2} U_{11}^{(2)} V+K_{1}^{*} K_{1}-K_{1}^{*} U_{11}^{(2)^{*}} U_{11}^{(2)} K_{1}\right] U_{21}^{(2)} U_{21}^{(2)} \widetilde{d}
\end{aligned}
$$

Similar to [42] one can easily prove that for any $\widetilde{b}_{\perp} \in \widetilde{\mathcal{B}}^{\perp}$ and $\widetilde{c}_{\perp} \in \widetilde{\mathcal{C}}^{\perp}$ the following relations hold:

$$
\begin{aligned}
& K_{1}^{*} K_{1} \widetilde{c}_{\perp}=\widetilde{c}_{\perp}+U_{22}^{(1)} K_{2} U_{11}^{(2)} V \widetilde{c}_{\perp}+V^{*} U_{11}^{(2)^{*}} K_{2}^{*} U_{22}^{(1)^{*}} \widetilde{c}_{\perp} \\
& +V^{*} U_{11}^{(2) *} K_{2}^{*} U_{22}^{(1)^{*}} U_{22}^{(1)} K_{2} U_{11}^{(2)} V \widetilde{c}_{\perp}
\end{aligned}
$$

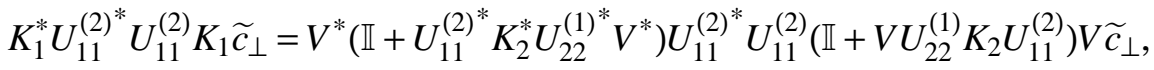

$$
\begin{aligned}
& K_{2}^{*} K_{2} \widetilde{b}_{\perp}=\widetilde{b}_{\perp}+K_{2}^{*} U_{22}^{(1)^{*}} V^{*} U_{11}^{(2)}+U_{11}^{(2)} V U_{22}^{(1)} K_{2} \widetilde{b}_{\perp} \\
& +K_{2}^{*} U_{22}^{(1)^{*}} V^{*} U_{11}^{(2)^{*}} U_{11}^{(2)} V U_{22}^{(1)} K_{2} \widetilde{b}_{\perp} \text {. }
\end{aligned}
$$

Inserting these relations in (A.11) with the choice $\widetilde{c}_{\perp}=U_{21}^{(2)}{ }^{*} U_{21}^{(2)} \widetilde{d}$ and $\widetilde{b}_{\perp}=U_{11}^{(2)} V \widetilde{c}_{\perp}$ we obtain that it vanishes thus completing the proof of the first relation in (A.1). The proof of the second relation in (A.1) is similar and will therefore be omitted.

\section{REFERENCES}

[1] T. Aktosun, A factorization of the scattering matrix for the Schrödinger equation and for the wave equation in one dimension, J. Math. Phys. 33, 3865 - 3869 (1992).

[2] S. Albeverio, F. Gesztesy, R. Høegh-Krohn, and H. Holden, Solvable Models in Quantum Mechanics, Springer, Berlin, 1988.

[3] S. Albeverio, L. Dabrowski, and P. Kurasov, Symmetries of Schrödinger operators with point interactions, Lett. Math. Phys. 45, 33 - 47 (1998).

[4] C. Allard and R. Froese, A Mourre estimate for a Schrödinger operator on a binary tree, Rev. Math. Phys. (to appear); available from http: / /www. ma. utexas.edu/mp_arc/ as 98-497.

[5] Y. Avishai and J.M. Luck, Quantum percolation and ballistic conductance on a lattice of wires, Phys. Rev. B 45, 1074 - 1095 (1992).

[6] J. Avron, A. Raveh, and B. Zur, Adiabatic quantum transport in multiply connected systems, Rev. Mod. Phys. 60, 873 - 915 (1988).

[7] J. Avron, P. Exner, and Y. Last, Periodic Schrödinger operators with large gaps and Wannier-Stark ladders, Phys. Rev. Lett. 72, 896 - 899 (1994).

[8] C.W.J. Beennakker, Random theory of quantum transport, Rev. Mod. Phys. 69, 731 - 808 (1997).

[9] B. Bollobás, Modern Graph Theory, Springer, New York, 1998.

[10] M. Büttiker, Four-terminal phase coherent conductance, Phys. Rev. Lett. 57, 1761 - 1764 (1986).

[11] M. Büttiker, Symmetry of electrical conduction, IBM J. Res. Develop. 32, 317 - 334 (1988).

[12] M. Büttiker, Transmission, reflection and the resistance of small conductors, in J.M. Chamberlain, L. Eaves, and J.-C. Portal (Eds.) Electronic Properties of Multilayers and Low-Dimensional Semiconductors Structures, NATO ASI Series B: Physics, Vol. 231, Plenum Press, New York, 1990, p. 51 - 73.

[13] R. Carlson, Hill's equation for a homogeneous tree, Electronic J. Diff. Eq., No. 23, (1997); available from http: //ejde.math. swt.edu.

[14] R. Carlson, Adjoint and self-adjoint differential operators on graphs, Electronic J. Diff. Eq., No. 6, (1998); available from http://ejde.math. swt.edu. 
[15] R. Carlson, Inverse eigenvalue problems on directed graphs, Trans. Amer. Math. Soc. 351, 4069 - 4088 (1999).

[16] M. Carreau, Four-parameter point-interaction in 1D quantum systems, J. Phys. A: Math. Gen. 26, 427 - 432 (1993).

[17] F. Chung, Spectral Graph Theory, Amer. Math. Soc, Providence, R.I., 1997.

[18] D. Cvetcovic, M. Doob, and H. Sachs, Spectra of Graphs, Academic Press, New York, 1979.

[19] H.L. Cycon, R.G. Froese, W. Kirsch, and B. Simon, Schrödinger Operators, Springer, Berlin, 1987.

[20] S. Datta, Electronic Transport in Mesoscopic Systems, Cambridge University Press, Cambridge, 1995.

[21] P. Deift and E. Trubowitz, Inverse scattering on the line, Commun. Pure Appl. Math. 32, 121 - 251 (1979).

[22] O.N. Dorokhov, Transmission coefficient and the localization length of an electron in $N$ bound disordered chains, JETP Lett. 36, 318 - 321 (1982)

[23] O.N. Dorokhov, Electron localization in a multichannel conductor, Sov. Phys. JETP 58, 606 - 615 (1983).

[24] O.N. Dorokhov, On the coexistence of localized and extended electronic states in the metallic phase, Solis State Commun. 51, 381 - 384 (1984).

[25] O.N. Dorokhov, Solvable model of multichannel localization, Phys. Rev. B 37, 10526 - 10541 (1988).

[26] B.A Dubrovin, A.T. Fomenko, and S.P. Novikov, Modern Geometry - Methods and Applications. Part I: The Geometry of Surfaces, Transformation Groups, and Fields, Springer, New York, 1991.

[27] P. Exner, Contact interactions on graph superlattices, J. Phys. A: Math. Gen. 29, 87 - 102 (1996).

[28] P. Exner and M. Tater, Evanescent modes in a multiple scattering factorization, Czech. J. Phys. 48, 617 - 624 (1998).

[29] L.D. Faddeev, Properties of the S-matrix of the one dimensional Schrödinger equation, Amer. Math. Soc. Transl. Ser. (2) 65, 139 - 166 (1967).

[30] F.R. Gantmacher, Matrizenrechnung, Vol. 1, Deutscher Verlag der Wissenschaften, Berlin, 1970.

[31] N.I. Gerasimenko and B.S. Pavlov, Scattering problems on compact graphs, Theor. Math. Phys. 74, 230 - 240 (1988).

[32] N.I. Gerasimenko, The inverse scattering problem on a noncompact graph, Teor. Mat. Fiz. 75, 187 - 200 (1988) (Russian).

[33] I.C. Gohberg and M.G. Krein, Introduction to the Theory of Linear Nonselfadjoint Operators in Hilbert Space, Amer. Math. Soc. Transl. of Math. Monographs Vol. 18, Providence, R.I., 1969.

[34] M.S. Harmer, Hermitian symplectic geometry and the Schrödinger operator on the graph, preprint (2000).

[35] R.A. Horn and C.R. Johnson, Topics in Matrix Analysis, Cambridge University Press, Cambridge, 1991.

[36] T. Kato, Perturbation Theory for Linear Operators, Springer, Berlin, 1966.

[37] J. Kellendonk, Noncommutative geometry of tilings and gap labeling, Rev. Math. Phys. 7, 1133 - 1180 (1995).

[38] V. Kostrykin, The Spectral Shift Function and Its Applications to Random Schrödinger Operators, Habilitationsschrift, RWTH Aachen, 1999 (unpublished).

[39] V. Kostrykin and R. Schrader, Cluster properties of one particle Schrödinger operators, Rev. Math. Phys. 6, 833 - 853 (1994).

[40] V. Kostrykin and R. Schrader, Cluster properties of one particle Schrödinger operators. II, Rev. Math. Phys. 10, 627 - 683 (1998).

[41] V. Kostrykin and R. Schrader, Scattering theory approach to random Schrödinger operators in one dimension, Rev. Math. Phys. 11, 187 - 242 (1999).

[42] V. Kostrykin and R. Schrader, Kirchhoff's rule for quantum wires, J. Phys. A: Math. Gen. 32, 595 - 630 (1999).

[43] V. Kostrykin and R. Schrader, One-dimensional disordered systems and scattering theory, preprint (1998); available from http: //www-sfb288. math.tu-berlin.de/abstractNew/337.

[44] V. Kostrykin and R. Schrader, The density of states and the spectral shift density of random Schrödinger operators, Rev. Math. Phys. 12 (2000) (to appear); available from http: / /www.ma.utexas.edu/mp_arc/ as $99-239$.

[45] V. Kostrykin and R. Schrader, Kirchhoff's rule for quantum wires. II: The inverse problem with possible applications to quantum computers, Fortschritte der Physik 48 (2000) (to appear); available from http: / / xxx.uni-augsburg.de/abs/quant-ph/9910053.

[46] V. Kostrykin and R. Schrader, Global bounds for the Lyapunov exponent and the integrated density of states of random Schrödinger operators in one dimension, preprint (2000); available from http: / / www . ma . utexas .edu/mp_arc/ as 00-226.

[47] D. Kowal, U. Sivan, O. Entin-Wohlman, and Y. Imry, Transmission through multiply-connected wire systems, Phys. Rev. B 42, 9009 - 9018 (1990).

[48] P. Kuchment and H. Zeng, Convergence of spectra of mesoscopic systems collapsing onto a graph, preprint (2000), available from http: / / www . ma . utexas .edu/mp_arc/ as 00-308.

[49] P. Kurasov, Distribution theory for discontinuous test functions and differential operators with generalized coefficients, J. Math. Anal. Appl. 201, 297 - 323 (1996).

[50] P. Kurasov and B.S. Pavlov, Micro spectral properties of crystals and their band structure, p. 259 -264 in M. Fannes, C. Maes, and A. Verbeure (Eds.) On Three Levels, Plenum, New York, 1994. 
[51] R. Landauer, Electrical resistance of disordered one-dimensional lattices, Philos. Mag. 21, 863 - 867 (1970).

[52] I.M. Lifshitz, S.A. Gredeskul, and L.A. Pastur, Theory of the passage of particles and waves through randomly inhomogeneous media, Sov. Phys. JETP 56, 1370 - 1378 (1982).

[53] I.M. Lifshitz, S.A. Gredeskul, and L.A. Pastur, Introduction to the Theory of Disordered Systems, Wiley, New York, 1988.

[54] A.V. Marchenko and L.A. Pastur, Transmission of waves and particles through long random barriers, Theor. Math. Phys. 68, 929 - 940 (1986).

[55] P.A. Mello, P. Pereyra, and N. Kumar, Macroscopic approach to multichannel disordered conductors, Ann. Phys. 181, 290 - 317 (1988).

[56] S.P. Novikov, Schrödinger operators on graphs and symplectic geometry, p. $397-413$ in E. Bierstone, B. Khesin, A. Khovanskii, and J. Marsden (Eds.), The Arnoldfest (Proceedings of a Conference in Honour V.I. Arnold for His Sixtieth Birthday, Fields Institute Communications Series, Vol. 24, American Mathematical Society, Providence, R.I., 1999.

[57] R. Redheffer, Difference equations and functional equations in transmission-line theory, in E. F. Beckenbach (Ed.) Modern Mathematics for the Engineer, New York, McGraw-Hill, 1961.

[58] R. Redheffer, On the relation of transmission line theory to scattering and transfer, J. Mathematics and Physics 41, 1 - 41 (1962).

[59] M. Reed and B. Simon, Methods of Modern Mathematical Physics, II: Fourier Analysis, Self-Adjointness, Academic Press, New York, 1975.

[60] M. Reed and B. Simon, Methods of Modern Mathematical Physics, III: Scattering Theory, Academic Press, New York, 1979.

[61] M. Reed and B. Simon, Methods of Modern Mathematical Physics, IV: Analysis of Operators, Academic Press, New York, 1978.

[62] M. G. Rozman, P. Reineker, and R. Tehver, One dimensional scattering: Recurrence relations and differential equations, Phys. Rev. A 49, 3310 - 3321 (1994).

[63] J. Rubinstein and M. Schatzman, Variational problems on multiply connected thin strips. I: Basic estimetes and convergence of the Laplacian spectrum, Preprint (1999).

[64] M. Sassoli de Bianchi and M. Di Ventra, On the number of states bound by one-dimensional finite periodic potentials, J. Math. Phys. 36, 1753 - 1764 (1995).

[65] M. Sassoli de Bianchi and M. Di Ventra, Differential equations and factorization property for the onedimensional Schrödinger equation with position-dependent mass, Europ. J. Phys. 16, 260 - 265 (1995).

[66] Y. Saito, Convergence of the Neumann Laplacians on shrinking domains, Analysis (to appear).

[67] Y. Saito, The limiting equation for Neumann Laplacians on shrinking domains, Electronic J. Diff. Eq., No. 31, (2000); available from http://ejde.math.swt.edu.

[68] P. Šeba, The generalized point interaction in one dimension, Czech. J. Phys. 36, 667 - 673 (1986).

[69] A.D. Stone, P.A. Mello, K. Muttalib, and J.-L. Pichard, Random matrix theory and maximum entropy models for disordered conductors, p. 369 - 448 in B.L. Altschuler, P.A. Lee, and R.A. Webb (Eds.) Mesoscopic Phenomena in Solids, North Holland, Amsterdam, 1991.

[70] G. Strang, Linear Algebra and its Applications, Academic Press, New York, 1980.

[71] B.Y. Tong, Electronic structure of one-dimensional binary alloys, Phys. Rev. 175, 710 - 722 (1968).

[72] D.R. Yafaev, Mathematical Scattering Theory. General Theory, Amer. Math. Soc. Transl. of Math. Monographs Vol. 105, Providence, RI, 1992.

VAdim Kostrykin, Fraunhofer-Institut FÜr LASERTEChniK, Steinbachstrasse 15, D-52074, AACHEN, GERMANY

E-mail address: kostrykindt-online.de, kostrykin@ilt.fhg.de

Robert Schrader, Institut FÜr Theoretische Physik, Freie UniVERsität Berlin, ARnimalleE 14, D-14195 BERLIN, GERMANY

E-mail address: schraderephysik. fu-berlin.de 